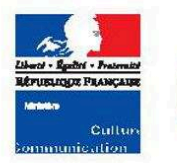

Secrétariat général

Service de la

politiques culturelles

et de l'innovation

Département

des études,

de la prospective
et des statistiques

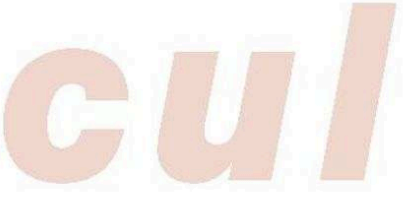

82, rue Saint-Honoré, 75033 Paris cedex 0

풍 0140157917 - 国 0140157999

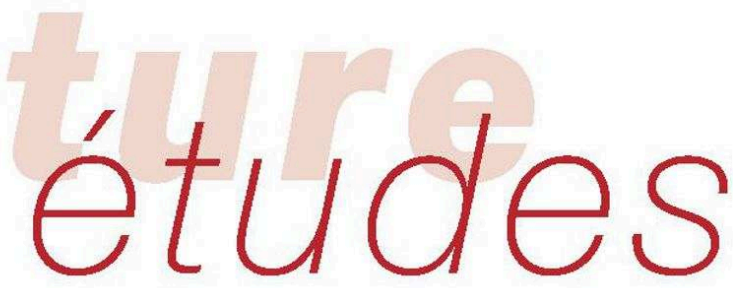

\title{
La diversité culturelle dans l'industrie de la musique enregistrée en France (2003-2008)
}

Marc Bourreau**, François Moreau* et Pierre Senellart**

Avant-propas

Cultural diversity within the French recorded music

La diversité culturelle est aujourd'hui un objectif central des politiques publiques culturelles, en particulier depuis l'adoption de la Convention sur la protection et la promotion de diversité des expressions culturelles de l'Unesco en 2005, en vigueur depuis le 18 mars 2007 après sa ratification par la France et l'Union européenne. De nombreuses mesures de politique culturelle s'y référent, notamment dans les secteurs des industries culturelles. Préoccupation principalement politique, la diversite culturelle n en possede pas moins des dimensions economiques car elle renvole a des questions importantes - concurence, cancentration inaustialle, pouvoir de ailleurs dans le contexte actuel renouvelées par une plo balisation économique et les effets produits par la revolution numérique.

Conscient des enjeux de politique publique que pose la question de la diversité, le Secrétariat général l'a inscrit, depuis plusieurs annes, dans son programme de recherche'. En 2008 , it a lancé un appel a propositions de recherche sur "l'appréciation et les processus de modification de la diversite dans les fitieres dindustrie culturelle $*$ Visant à enrichir l'ensemble des résultats de l'analyse socioéconomique sur le sujet, l'appel invitait les chercheurs à apporter des elements de réponse quant à l'appreciation des différentes dimensions de la diversité culturelle (défintitions et mesures) et l'identification des processus par lesquels la diversité des produits culturels offerts, distribués et consommés a pu se modifien.

Alors que l'industrie musicale française subit une crise et a vu les ventes de supports disques s'effondrer au cours des annees 2000 , l'étude de l'evolution de la diversité sur sur la réalité d'un effet "longue traine " pour le marché sur la realte a un effet "longue traine "pour le marche
examine. industry (2003-2008)

Le marché du disque est réputé pour être très concentré sur un petit nombre de firmes, les quatre majors réalisent en effet les trois quarts des ventes, alors que plusieurs centaines de labels indépendants se partagent le reste du marché. De plus, l'industrie de la musique enregistrée s'inscrit dans la logique du modèle éditorial. En raison de faibles coûts fixes de production et de l'incertitude entourant le succès d'une œuvre donnée, le nombre de variétés produites est très élevé et quelques succès permettent de compenser les nombreux échecs commerciaux. Après une décennie 1990 florissante, l'industrie de la musique enregistrée s'est, depuis 2003, enfoncée dans la crise. Sans que l'on cherche ici à identifier l'ampleur ni même l'existence d'une causalité entre les deux phénomènes, la crise des ventes de musique enregistrée s'inscrit en parallèle d'un processus avancé de numérisation de la filière. En matière de diversité, l'industrie de la musique enregistrée offre donc un éclairage spécifique : comment évolue la diversité culturelle dans une industrie en crise? Comment les ventes en ligne influent-elles sur la diversité ? Le poids conjugué très important de la grande distribution alimentaire et des grandes surfaces spécialisées dans les produits culturels dans les ventes de musique enregistrée a-t-il des conséquences en matière de diversité ?

Une analyse de la base de données compilée par l'institut GfK sur les ventes de musique enregistrée en France sur la période 2003-2008 (voir «Les données», p. 14) permet d'apporter des éléments de réponse à la question de la diversité culturelle, à partir des critères de variété, d'équilibre et de disparité (voir «Éléments de méthodologie », p. 14).

* Laboratoire Information, Coordination, Incitations (ICI), Université de Bretagne occidentale; ** Télécom ParisTech.

1. Voir notamment Tristan MATTELART, Enjeux intellectuels de la diversité culturelle. Eléments de déconstruction théorique, Paris, Ministère de la Culture et de la Communication, DEPS, coll. "Culture prospective », 2009-2, juillet 2009. Par ailleurs, cette réflexion sur la diversité culturelle comprend également un volet consacré à la promotion du dialogue interculturel, voir Hélène HATZFELD, Vincent BillereY, Repères pour un dialogue interculturel, Ministère de la Culture et de la Communication, Secrétariat général (SCPC/DREST), 2010, en ligne sur www.culture.gouv.fr/ rubrique Politiques ministérielles 2. L'analyse se limite au marché des ventes physiques de musique enregistrée (albums en format CD), à l'exclusion du marché du DVD musical, et ne recouvre donc pas le marché numérique via l'internet ou la téléphonie mobile (vente au titre, abonnement, streaning, sonneries, etc.). Toutefois, sur la période considérée, ces deux marchés restent marginaux. Ainsi, en 2008, selon GfK, le marché du DvD musical ne représentait que 7,5\% du marché physique de la musique enregistrée et le marché numérique ne comptait que pour $5,9 \%$ des ventes en valeur de musique enregistrée en France. Voir www.disqueenfrance.com

Directeur de publication: Jean-François Chaintreau, chef du service de la coordination des politiques culturelles

et de l'innovation, en charge du département des études, de la prospective et des statistiques.

$2011-5$ - octobre 2011

Responsable des publications: Edwige Millery 


\section{La diversité culturelle dans l'industrie du livre en France (2003-2007)}

Cultural Diversity in the French Book Publishing Industry (2003-2007)

\section{François Moreau et Stéphanie Peltier}

Éditeur : Département des études, de la prospective et des statistiques

Lieu d'édition : Paris

Année d'édition : 2011

Date de mise en ligne : 8 juillet 2015

Collection : Culture études

ISBN électronique : 9782111398986

\section{Sbooks}

http://books.openedition.org

\section{Édition imprimée}

Date de publication : 1 octobre 2011

Nombre de pages : 16

\section{Référence électronique}

MOREAU, François ; PELTIER, Stéphanie. La diversité culturelle dans l'industrie du livre en France

(2003-2007). Nouvelle édition [en ligne]. Paris : Département des études, de la prospective et des statistiques, 2011 (généré le 25 avril 2021). Disponible sur Internet : <http://books.openedition.org/ deps/200>. ISBN : 9782111398986 

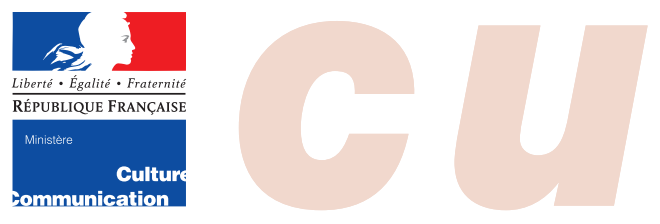

Secrétariat général

Service de la

coordination des

politiques culturelles

et de l'innovation

Département

des études,

de la prospective

et des statistiques

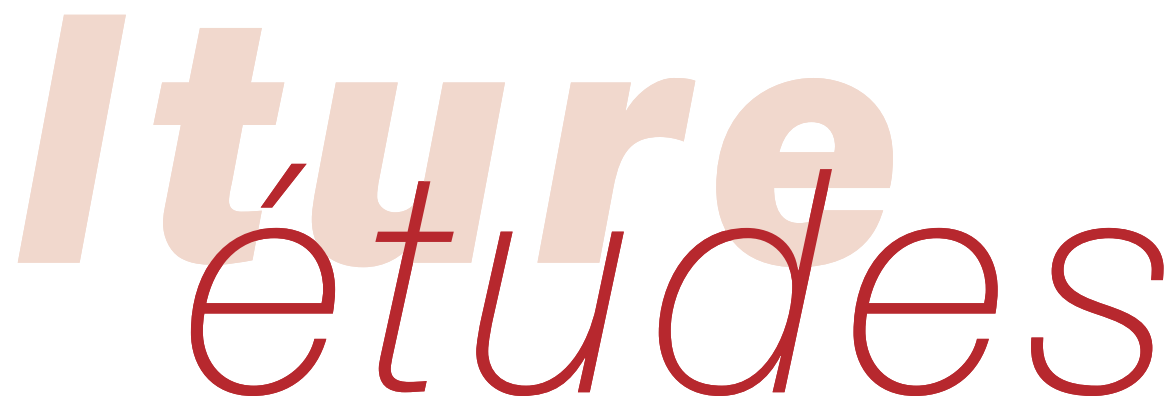

182, rue Saint-Honoré, 75033 Paris cedex 01

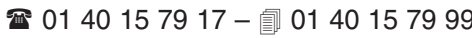

\title{
La diversité culturelle dans l'industrie du livre en France (2003-2007)
}

\author{
François Moreau* et Stéphanie Peltier** \\ Cultural Diversity in the French Book Publishing Industry \\ (2003-2007)
}

\section{Avant-propos}

La diversité culturelle est aujourd'hui un objectif central des politiques publiques culturelles, en particulier depuis l'adoption de la Convention sur la protection et la promotion de diversité des expressions culturelles de l'Unesco en 2005, en vigueur depuis le 18 mars 2007 après sa ratification par la France et l'Union européenne. De nombreuses mesures de politique culturelle s'y réfèrent, notamment dans les secteurs des industries culturelles. Préoccupation principalement politique, la diversité culturelle n'en possède pas moins des dimensions économiques car elle renvoie à des questions importantes - concurrence, concentration industrielle, pouvoir de marché et optimalité économique - qui se trouvent par ailleurs, dans le contexte actuel, renouvelées par une globalisation économique et les effets produits par la révolution numérique.

Conscient des enjeux de politique publique que pose la question de la diversité, le Secrétariat général l'a inscrit, depuis plusieurs années, dans

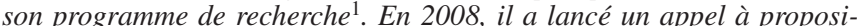
tions de recherche sur "l'appréciation et les processus de modification de la diversité dans les filières d'industrie culturelle». Visant à enrichir l'ensemble des résultats de l'analyse socioéconomique sur le sujet, l'appel invitait les chercheurs à apporter des éléments de réponse quant à l'appréciation des différentes dimensions de la diversité culturelle (définitions et mesures) et l'identification des processus par lesquels la diversité des produits culturels offerts, distribués et consommés a pu se modifier.

Dans le cadre de l'édition de livres, un secteur qui se caractérise en France par sa concentration, et alors que la question de la surproduction est régulièrement posée, l'étude de l'évolution de la diversité au cours des années 2000 apporte notamment des éléments de réponse sur l'efficience du dispositif législatif - loi sur le prix unique du livre - dans sa capacité à préserver l'existence d'une diversité des titres et de leur distribution, et vient conforter, tout en la nuancant, l'hypothèse de la validité du modèle économique de la longue traîne.

Jean-François CHAINTREAU
La production éditoriale française a considérablement progressé au cours des années 2000, au point que la question de la surproduction est désormais posée à l'occasion de chaque rentrée littéraire. Pour autant, l'offre, réputée prolifique, se traduit-elle par une plus grande diversité de consommation ou, comme le dénoncent certains, ne conduit-elle pas la demande à se concentrer davantage sur quelques best-sellers et auteurs stars? La préservation de la diversité de la création et de la diffusion fut au fondement de la loi sur le prix unique du livre (seul un rabais de $5 \%$ sur le prix de vente au public fixé par l'éditeur est autorisé) votée en France en 1981, dans le but de préserver un réseau dense et diversifié de détaillants (petites librairies, mais aussi grands indépendants à larges assortiments), dont l'existence paraissait menacée par les pratiques de discount $^{2}$.

Cet objectif a-t-il été atteint? La diversité offerte et consommée est-elle singulièrement différente dans les petites et grandes librairies par rapport à la grande distribution alimentaire et aux grandes surfaces spécialisées? Enfin, au tournant d'une révolution, sans précédent depuis

\footnotetext{
* Laboratoire Information, Coordination, Incitations (ICI), Université de Bretagne occidentale ; ** Laboratoire de recherche management, organisation et sociétés (LR-MOS), université de La Rochelle et Groupe de recherche angevin en économie et management (Granem), université d'Angers. Les auteurs remercient les membres du comité de pilotage réuni par le ministère de la Culture et de la Communication/DEPS, tout particulièrement Yann Nicolas, Hervé Renard et François Rouet, pour leurs commentaires avisés sur le rapport dont est issu cet article et Sarah Machat (LR-MOS, université de La Rochelle) pour sa contribution au traitement statistique.

1. Voir notamment Tristan MatTelart, Enjeux intellectuels de la diversité culturelle. Éléments de déconstruction théorique, Paris, Ministère de la Culture et de la Communication, DEPS, coll. «Culture prospective», 2009-2, juillet 2009. Par ailleurs, cette réflexion sur la diversité culturelle comprend également un volet consacré à la promotion du dialogue interculturel, voir Hélène HATZFELD, Vincent BILLEREY, Repères pour un dialogue interculturel, Ministère de la Culture et de la Communication, Secrétariat général (SCPCI/DREST), 2010, en ligne sur www.culture.gouv.fr/rubrique Politiques ministérielles.

2. Pour un rappel des objectifs de la loi, voir Hervé GaYMARD, Situation du livre. Évaluation de la loi relative au prix du livre et questions prospectives, rapport à la ministre de la Culture et de la Communication, mars 2009. Voir aussi H. GAYMARD, Pour le livre, Paris, La Documentation française/Gallimard, 2009.
} 
l'avènement de l'imprimerie, la question de l'impact du numérique sur la diversité culturelle se pose. Favorise-t-il un renforcement des ventes des best-sellers ou prolonget-il la vie de livres à faibles tirages conformément à la théorie de la longue traîne formulée par Chris Anderson ${ }^{3}$ ?

La diversité culturelle est analysée au regard de trois dimensions : l'ampleur du nombre de livres produits et consommés (la variété), le degré d'inégalité dans la répartition des ventes entre les différents titres (l'équilibre), le degré de disparité entre les différents titres vendus en s'intéressant à leurs caractéristiques propres (auteur, éditeur) (la disparité). Le champ d'analyse se focalise sur trois segments de marché : la littérature, la bande dessinée et les livres pour la jeunesse ${ }^{4}$, et s'appuie sur une exploitation inédite des données des ventes mensuelles de livres «sortie de caisse » compilées par l'institut GfK sur la période 2003-2007 (voir «Les données », p. 14).

\section{CONTEXTE $^{5}$}

\section{Principales caractéristiques du marché du livre et leur évolution, 2003-2007}

Entre 2003 et 2007, le chiffre d'affaires cumulé des trois segments retenus a connu une croissance relativement soutenue $(+11,5 \%$ sur l'ensemble de la période, soit une croissance annuelle moyenne de $2,8 \%$ ) (graphique 1). Toutefois, une rupture de tendance est apparue à partir de 2005 avec une croissance limitée à $2 \%$ les deux dernières années. Ce ralentissement de la croissance du chiffre d'affaires se cumule d'ailleurs avec un tassement puis une légère baisse des volumes vendus. D'environ 193 millions d'unités en 2004, les ventes de livres des trois segments analysés sont tombées à 189 millions pour l'année 2007, soit une baisse de $2 \%$. La catégorie littérature représente sur toute la période environ la moitié des exemplaires vendus, les livres pour la jeunesse comptant pour $30 \%$ et les bandes dessinées pour $20 \%$. L'évolution des ventes est assez similaire pour les trois segments : une légère croissance en début de période, suivie d'un tassement puis d'une légère baisse vers la fin de la période.

Depuis 2007, les grandes surfaces alimentaires (GSA) et les grandes surfaces spécialisées (GSs), comme les Fnac ou les magasins Virgin, représentent chacune environ $27 \%$ des volumes de livres vendus sur les trois segments étudiés.
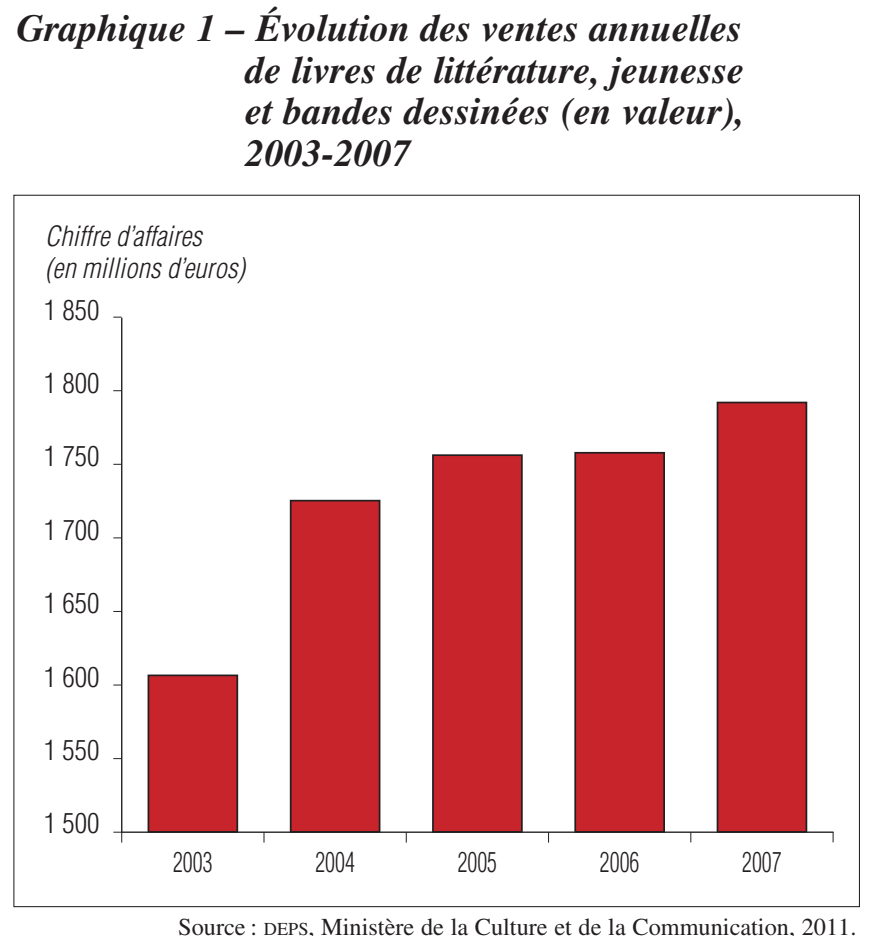
Graphique 2 - Évolution de la répartition des ventes mensuelles par circuit de distribution (en volume)

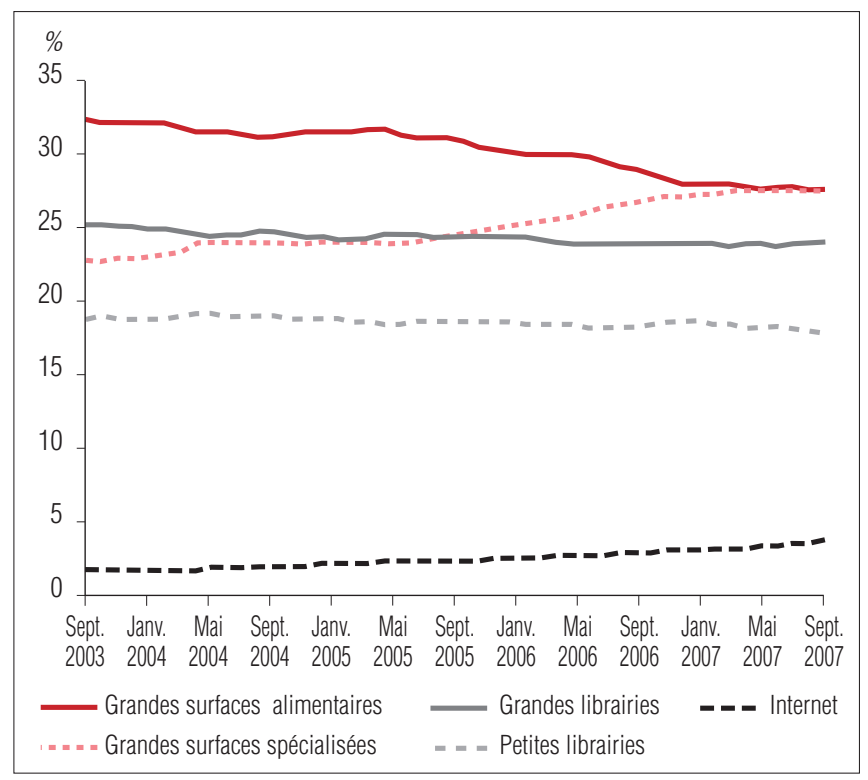

Source : DEPS, Ministère de la Culture et de la Communication, 2011.

3. Chris ANDERSON, "The Long Tail", Wired, octobre 2004 ; id., The Long Tail, Londres, Random House, 2006.

4. Sur des marchés comme celui de la musique enregistrée ou des films en vidéo, tous les produits peuvent être considérés comme plus ou moins substituables. Tout au moins, répondent-ils tous à la satisfaction d'un même besoin pour les consommateurs, celui de se divertir. Cette hypothèse est plus délicate pour le livre. Le marché du livre peut se scinder en quatre segments à partir d'une double opposition ayant trait à la décision d'achat (distinction entre livres prescrits de manière formelle ou non prescrits) et à la lecture (distinction entre livres de consultation et autres livres). Aussi, l'analyse s'est centrée sur les livres non prescrits essentiellement destinés à la lecture de loisir, principalement la littérature, la bande dessinée et les livres pour la jeunesse. Le premier est intéressant car, s'il ne représente que $20 \%$ des ventes de livres, il n'en reste pas néanmoins le premier segment du marché. La bande dessinée est un marché en forte expansion avec, qui plus est, une forte présence en ligne, notamment via des sites d'informations et de conseils spécialisés. Enfin, les livres pour la jeunesse, secteur également en expansion depuis plusieurs années, s'adressent, en partie, à un public massivement connecté à l'internet et, là aussi, les analyses comparées de l'évolution de la diversité peuvent être riches d'enseignements.

5. Comme pour la plupart des produits culturels, les ventes de livres font l'objet d'une forte saisonnalité. Les ventes du mois de décembre sont systématiquement de deux à trois fois plus importantes que les ventes moyennes des autres mois de l'année. Au sein du segment de la littérature, la fiction moderne présente une composante saisonnière accrue puisqu'en fin d'année se cumulent la rentrée littéraire et la période des fêtes. En règle générale, les données désaisonnalisées sont utilisées afin de faciliter la lecture des graphiques et l'identification de tendances d'évolution des différentes variables étudiées. La méthode de désaisonnalisation retenue est la méthode des moyennes mobiles sur 12 mois. 
La part des grandes librairies se monte à environ $24 \%$, contre $17 \%$ pour les petites librairies et $4 \%$ pour les ventes en ligne (graphique 2). Contrairement aux évolutions par segment, les évolutions par circuit de distribution ont été assez marquées sur la période. Ainsi, l'essentiel de la baisse des ventes de livres observée depuis 2005 est imputable à la GSA. Les ventes en librairies (petites ou grandes) ont très légèrement baissé, alors que dans le même temps les ventes sur l'internet se sont lentement développées, même si la part de marché de ce circuit de distribution reste encore inférieure à $5 \%$. En revanche, la baisse des ventes en GSA a été massive (une chute de près de $15 \%$ entre 2005 et 2007) alors que dans le même temps les ventes en GSs n'ont cessé de progresser (la hausse dépasse $20 \%$ sur l'ensemble de la période). Ainsi, alors que jusqu'au début 2007, la GSA restait, en volume, le circuit de distribution privilégié pour les trois segments étudiés, la GSS a pris de justesse, fin 2007, la première place. Toutefois, si l'on agrège les petites et grandes librairies, c'est bien ce circuit « librairie » qui apparaît comme dominant avec une part de marché d'environ $40 \%$.

L'analyse du budget moyen de l'achat d'un livre sur ces différents circuits permet d'affiner cette analyse (graphique 3). Le faible budget alloué par livre en GSA explique qu'en termes de chiffre d'affaires, elle ne soit que le troisième circuit de distribution (avec une part de marché d'environ $23 \%$ en 2007), derrière les grandes librairies (envi-

\section{Graphique 3 - Évolution du budget moyen d'achat d'un livre selon les circuits de distribution}

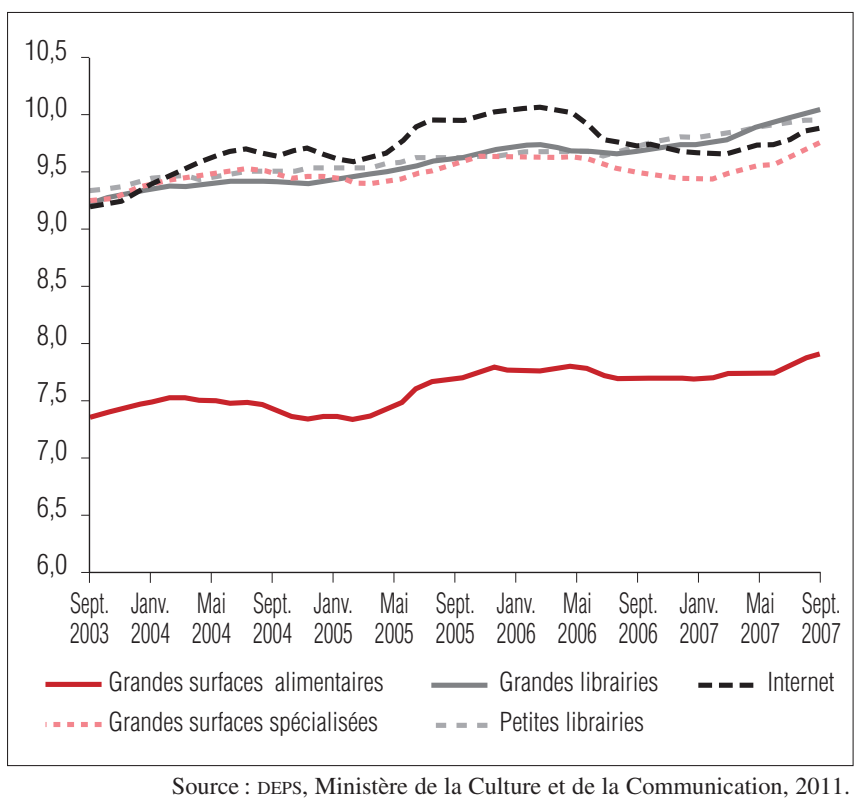

ron $25 \%$ du marché en valeur en 2007) et surtout les GSS (environ $29 \%$ ). L'écart entre le budget moyen de vente d'un livre en GSA par rapport aux autres circuits tient vraisemblablement à la structure des ventes dans ce circuit particulier. D'une part, les ventes de livres pour la jeunesse y sont plus importantes car elles relèvent souvent de l'achat d'impulsion, d'autant plus si des enfants sont présents durant la fréquentation d'un magasin de la GSA. D'autre part, la GSA affectionne les ventes d'ouvrages soldés ou à prix éditeur réduit.

Ainsi, pour les trois catégories étudiées sur la période 2003-2007, le marché du livre est relativement stable, notamment au regard d'autres marchés de biens culturels comme la musique enregistrée ou la vidéo; leurs parts de marché respectives restent stables sur la période, les ventes en GSA se réduisant au profit de celles enregistrées en GSS et des ventes en ligne.

\section{LA DIVERSITÉ A-T-ELLE AUGMENTÉ SUR LA PÉRIODE 2003-2007 ${ }^{6}$ ?}

La diversité s'accroit avec le nombre de titres (variété), avec l'uniformisation de la distribution des ventes entre ces titres (équilibre) et lorsqu'ils présentent un plus faible degré de standardisation (disparité). L'importance de la prise en compte simultanée de ces trois dimensions peut être restituée par rapport au débat récurrent : trop de livres tue-t-il le livre ? La plus grande variété d'ouvrages offerte permetelle aux consommateurs de trouver plus facilement des livres proches de leurs préférences ou est-elle « factice, constituant une sorte de décor pour la vente de quelques produits seulement ${ }^{7} »$ ? L'augmentation du nombre de livres édités peut en effet induire deux effets contraires sur la diversité consommée. Elle peut contribuer à son augmentation, si les consommateurs sont plus à même de trouver des livres correspondant à leurs goûts. À l'inverse, l'augmentation de la production littéraire peut réduire la diversité consommée en raison de la plus grande difficulté à formuler des choix face à la surabondance de livres disponibles. Les consommateurs vont alors, au contraire, concentrer leurs achats sur un nombre restreint de titres ${ }^{8}$ en privilégiant ceux largement promus ou ceux ayant déjà fait l'objet d'expériences de consommation passées (confiance à un auteur ou à un éditeur pour ses choix éditoriaux). Le nombre (variété) irait alors de pair avec une concentration des ventes sur un nombre restreint de titres (faible équilibre) et une plus forte standardisation (faible disparité).

6. Ce travail s'appuie sur l'approche multidimensionnelle inspirée par Stirling reposant sur le triptyque «variété, équilibre, disparité ». Voir A. STIRLING, On the Economics and Analysis of Diversity, mimeo, SPRU Electronic, Working Paper, 1999, n 28 ; id., "A General Framework for Analysing Diversity in Science, Technology and Society", Journal of the Royal Society Interface, 2007, 4(15), p. 707-719.

7. Françoise BenHAMOU, Éléments de réflexion et propositions à propos de la diversité culturelle. Les cas du livre et du cinéma en salles. Rapport pour le Groupe d'analyse stratégique des industries culturelles (Gasic), Paris, Ministère de la Culture et de la Communication, 2008 , p. 3.

8. Ce phénomène fait référence à l'économie de l'attention et est connu en marketing sous le terme overchoice : voir H. A. SimoN, "Designing Organizations for an Information-Rich World", dans M. GreEnBERgER (ed.), Computers, Communication, and the Public Interest, Baltimore, The Johns Hopkins Press, 1971. 


\section{Variété}

La première dimension de la diversité est appréhendée à travers deux indicateurs : la variété produite et la variété consommée.

\section{Une variété produite en hausse...}

Pour estimer la variété produite, à partir des données fournies par GfK, le nombre total de titres qui ont connu leur semaine de première vente au cours d'une année donnée a été évalué ${ }^{9}$. Cette mesure de la variété produite se base sur l'hypothèse implicite que, sur les segments étudiés, un livre se vend systématiquement au moins une fois lors de la semaine de sa sortie. À l'exception des préventes qui demeurent marginales dans le livre, il est réaliste de considérer que la date de sortie d'un titre correspond donc à sa date de première vente.

La production éditoriale (tous segments confondus) enregistre une légère hausse. Le nombre de titres vendus au cours de la première semaine de commercialisation est passé de 23916 en 2003 à 25863 en 2007 soit une hausse d'environ $8 \%{ }^{10}$. Cette hausse n'a pas été continue sur la période. Après un léger fléchissement entre 2003 et 2004, le nombre de titres produits n'a cessé de croître jusqu'en 2006 mais a subi une légère contraction en 2007 $\left(-1,2 \%{ }^{11}\right)$.

Sans surprise, la littérature occupe la plus grande part du nombre de titres publiés dans l'ensemble des trois catégories éditoriales étudiées. Elle représente $54 \%$ des livres sortis en 2007 contre $27 \%$ pour les livres jeunesse et $19 \%$ pour les bandes dessinées. Cependant, c'est dans la bande dessinée que l'accroissement du nombre de références produites a été le plus élevé sur la période 2003-2007 $(+43 \%)$. Les livres jeunesse enregistrent une hausse de près de $8 \%$, alors que la production littéraire ne s'accroît que très légèrement $(+0,8 \%)$.

La hausse de la variété produite conduit-elle à une plus grande variété consommée ? Les données de la base GfK semblent plutôt valider cette hypothèse.

\section{... comme la variété consommée}

Toutes catégories confondues, le nombre de titres différents vendus mensuellement (la variété consommée, ou plus précisément achetée) suit une tendance continue à la hausse (tableau 1). En moyenne annuelle, le nombre de titres différents vendus par mois a connu une augmentation de l'ordre de $30 \%$. Il est même frappant de constater que la hausse de la variété consommée est beaucoup plus importante que celle de la variété produite, qui n'a progressé que de $8 \%$. Ainsi, l'augmentation de la variété consommée ne se focalise pas sur les nouveautés mais touche également des titres plus anciens. Ce phénomène peut résulter de l'impact de nouveaux modes de prescription (blogs, forums, etc.) qui incitent les consommateurs à diversifier leurs actes d'achat. À cela s'ajoute le développement des ventes en ligne autorisant l'achat de titres ne se trouvant pas ou difficilement dans les magasins traditionnels (livres parus depuis plusieurs années, livres à tirage très limité, livres étrangers, etc.). L'évolution de la variété consommée sur l'internet nous donne un élément de validation de cette hypothèse (voir infra).

L'analyse par genre révèle une croissance plus forte du nombre de titres différents vendus en bande dessinée, loin devant les livres jeunesse et la littérature dont la progression a été légèrement moins rapide que la moyenne.

De plus, la cohérence entre les évolutions de la variété produite et la variété consommée par genre apparaît flagrante. La hiérarchie reste en effet similaire entre les différents segments. La bande dessinée enregistre la plus forte progression de la variété produite $(+43 \%)$ de même que pour la variété consommée (+67\%). Les livres pour la jeunesse présentent le deuxième taux de progression tant pour la variété produite que pour la variété consommée (respectivement $+8 \%$ et $+32 \%$ ). La littérature est dernière selon les deux critères (respectivement $+0,8 \%$ et $+21 \%{ }^{12}$ ) .

Si l'on s'en tient à sa première dimension, la variété, la diversité s'accroît donc incontestablement. Tant le nombre de nouveaux titres mis sur le marché que ceux vendus sont orientés à la hausse sur tous les segments étudiés avec un

Tableau 1 - Nombre moyen de titres différents vendus mensuellement par an (2003-2007)

\begin{tabular}{|c|c|c|c|c|c|c|}
\hline & 2003 & 2004 & 2005 & 2006 & 2007 & $\begin{array}{c}\text { Variation 2003/2007 } \\
\text { (en \%) }\end{array}$ \\
\hline Littérature & 48260 & 51050 & 53450 & 56260 & 58240 & 21 \\
\hline Bandes dessinées & 11540 & 13480 & 15330 & 17210 & 19300 & 67 \\
\hline Livres jeunesse & 25320 & 27230 & 28820 & 31380 & 33420 & 32 \\
\hline Total & 85120 & 91760 & 97600 & 104850 & 110960 & 30 \\
\hline
\end{tabular}

Source : DEPS, Ministère de la Culture et de la Communication, 2011.

9. Malheureusement, les données disponibles auprès de GfK ne permettent pas d'évaluer la variété offerte, i.e. le nombre de références disponibles à un moment donné selon le circuit de distribution.

10. Cette augmentation tient essentiellement à la hausse des livres sortis en grand format (+ $18 \%$ sur la période 2003-2007), alors que le nombre de références produites en poche s'érode $(-12 \%)$.

11. La croissance du nombre de titres vendus pour la première fois peut tendre à être légèrement surévaluée par la méthode d'extrapolation des données de ventes utilisée par GfK (voir «Les données », p. 14).

12. L'écart est toujours d'une vingtaine de points. 
Tableau 2 - Part des titres et part des ventes pour la bande dessinée

\begin{tabular}{|lrrrrr|}
\hline $\begin{array}{l}\text { Part des titres } \\
\text { cumulant... }\end{array}$ & $\mathbf{2 0 0 3}$ & $\mathbf{2 0 0 4}$ & $\mathbf{2 0 0 5}$ & $\mathbf{2 0 0 6}$ & $\mathbf{2 0 0 7}$ \\
$50 \%$ des ventes & 3 & 3 & 3 & 3 & 3 \\
$40 \%$ des ventes & 22 & 21 & 20 & 20 & 20 \\
$9 \%$ des ventes & 34 & 34 & 34 & 36 & 37 \\
$1 \%$ des ventes & 41 & 42 & 43 & 41 & 40 \\
Total & $\mathbf{1 0 0}$ & $\mathbf{1 0 0}$ & $\mathbf{1 0 0}$ & $\mathbf{1 0 0}$ & $\mathbf{1 0 0}$ \\
\hline
\end{tabular}

Source : DEPS, Ministère de la Culture et de la Communication, 2011.

\section{Tableau 3 - Part des titres et part des ventes pour la jeunesse}

(en \%)

\begin{tabular}{|lrrrrr|}
\hline $\begin{array}{l}\text { Part des titres } \\
\text { cumulant... }\end{array}$ & $\mathbf{2 0 0 3}$ & $\mathbf{2 0 0 4}$ & $\mathbf{2 0 0 5}$ & $\mathbf{2 0 0 6}$ & $\mathbf{2 0 0 7}$ \\
$50 \%$ des ventes & 5 & 5 & 4 & 5 & 5 \\
$40 \%$ des ventes & 25 & 25 & 24 & 24 & 25 \\
$9 \%$ des ventes & 33 & 33 & 34 & 34 & 36 \\
$1 \%$ des ventes & 37 & 36 & 38 & 36 & 35 \\
Total & $\mathbf{1 0 0}$ & $\mathbf{1 0 0}$ & $\mathbf{1 0 0}$ & $\mathbf{1 0 0}$ & $\mathbf{1 0 0}$ \\
\hline
\end{tabular}

\section{Tableau 4-Part des titres et part des ventes pour la littérature}

\begin{tabular}{|lrrrrr|}
\hline $\begin{array}{l}\text { Part des titres } \\
\text { cumulant... }\end{array}$ & $\mathbf{2 0 0 3}$ & $\mathbf{2 0 0 4}$ & $\mathbf{2 0 0 5}$ & $\mathbf{2 0 0 6}$ & $\mathbf{2 0 0 7}$ \\
$50 \%$ des ventes & 2 & 2 & 1 & 1 & 1 \\
$40 \%$ des ventes & 15 & 15 & 14 & 14 & 14 \\
$9 \%$ des ventes & 33 & 33 & 34 & 35 & 36 \\
$1 \%$ des ventes & 50 & 50 & 51 & 50 & 49 \\
Total & $\mathbf{1 0 0}$ & $\mathbf{1 0 0}$ & $\mathbf{1 0 0}$ & $\mathbf{1 0 0}$ & $\mathbf{1 0 0}$ \\
\hline
\end{tabular}

Source : DEPS, Ministère de la Culture et de la Communication, 2011

effet plus marqué pour les bandes dessinées. L'offre pléthorique de livres, si souvent dénoncée, aurait au moins pour effet d'inciter à l'achat d'un plus grand nombre de titres différents. L'approfondissement du concept de diversité conduit cependant à nuancer ce constat.

\section{Équilibre}

L'augmentation de la variété consommée (nombre croissant de titres différents vendus) s'accompagne-t-elle d'une déconcentration des ventes sur un plus grand nombre de livres ou d'un renforcement des ventes de livres connaissant déjà le succès ?

\section{Une part de plus en plus restreinte de titres totalise la moitié des ventes}

Une plus forte polarisation des ventes sur une part restreinte de titres apparaît, quelle que soit la catégorie considérée. Moins de $5 \%$ des titres réalisent la moitié des ventes (tableaux 2, 3 et 4). La concentration semble légèrement s'accentuer avec le temps ${ }^{13}$. En moyenne annuelle, la part des titres réalisant la moitié des ventes est passée, entre 2003 et 2007, de 4,9\% à 4,6\% pour les livres pour la jeunesse, de $3,2 \%$ à $2,7 \%$ pour les bandes dessinées et de $1,7 \%$ à $1,2 \%$ pour la littérature. L'analyse de la proportion des titres réalisant $90 \%$ des ventes conduit au même constat : une tendance à la baisse pour la littérature et les bandes dessinées et une relative stabilité sur l'ensemble de la période pour les livres pour la jeunesse. Quel que soit le seuil considéré ( $50 \%$ ou $90 \%$ des ventes), la concentration apparaît donc plus élevée en littérature et plus faible en livres pour la jeunesse, la catégorie des bandes dessinées se trouvant dans une position intermédiaire.

Qu'en est-il des ventes des titres à faible tirage (ceux qui réalisent $1 \%$ des ventes), qui représentent ce que l'on dénomme la queue de la distribution? Quelle que soit la catégorie considérée, la part de ces titres, bien qu'importante, a tendance à diminuer entre 2003 et 2007 (tableaux 2,3 et 4). À l'inverse, une part croissante de titres réalise $9 \%$ des ventes : $37 \%$ pour la bande dessinée, $36 \%$ pour les livres pour la jeunesse et $36 \%$ pour la littérature (+ 3 points par rapport à 2003 pour chacune des catégories).

On note un léger renforcement de la concentration des ventes sur la tête (un nombre plus réduit de best-sellers réalisant au total la moitié des ventes), un creusement au milieu de la distribution (une plus grande proportion des titres se partage $9 \%$ des ventes) et une forme d'épaississement de la traîne (une part plus faible de titres à ventes épisodiques, bien qu'importante, se partage le dernier pourcentage des ventes ${ }^{14}$ ).

Toutefois, la légère diminution de la part des titres correspondant à une proportion donnée des ventes ne traduit pas nécessairement un accroissement des ventes de chacun des best-sellers. En effet, en raison de la forte croissance du nombre de titres différents vendus sur la période, elle peut masquer une augmentation du nombre de titres réalisant conjointement cette part des ventes, et donc finalement une réduction des ventes par titre.

\section{Une réduction de la part des titres à fortes ventes (plus de 1000 exemplaires par mois)}

Il est possible de classer les livres dans trois groupes distincts en fonction du nombre de ventes mensuelles : les titres à faibles ventes (moins de 100 exemplaires vendus

13. L'interprétation des évolutions doit se faire, surtout pour la catégorie jeunesse, en gardant à l'esprit que les valeurs mensuelles des moyennes mobiles sont assez sensibles à l'éventuelle sortie, sur la période d'un an entourant le mois considéré, d'un best-seller, comme les différents tomes de Harry Potter par exemple.

14. L'élargissement du panel en cours de période contribue à augmenter le nombre de titres à faibles ventes pris en compte (voir « Les données », p. 14). Si ce phénomène peut conduire à surestimer l'accroissement de la concentration des ventes sur la tête, il sous-estime à l'inverse l'effet d'épaississement de la traîne. 
par mois), les titres à ventes moyennes (entre 100 et 1000 exemplaires vendus par mois), les titres à fortes ventes (plus de 1000 exemplaires vendus) (graphique 4). Une tendance à la déconcentration des ventes apparaît. La part des titres à faibles ventes augmente de près de 4,5 points, passant de $75 \%$ des titres vendus en 2003 à près de $80 \%$ en 2007. Cette hausse s'effectue au détriment des livres à ventes moyennes qui représentent seulement $18 \%$ des titres vendus en 2007 contre $21 \%$ en 2003. La part des titres à fortes ventes enregistre une très légère érosion : reculant de 3,3\% des titres vendus mensuellement à 2,5\% entre 2003 et 2007 (soit - 0,8 point).

\section{Graphique 4-Distribution des titres par niveau de ventes (moyenne annuelle des données mensuelles)}

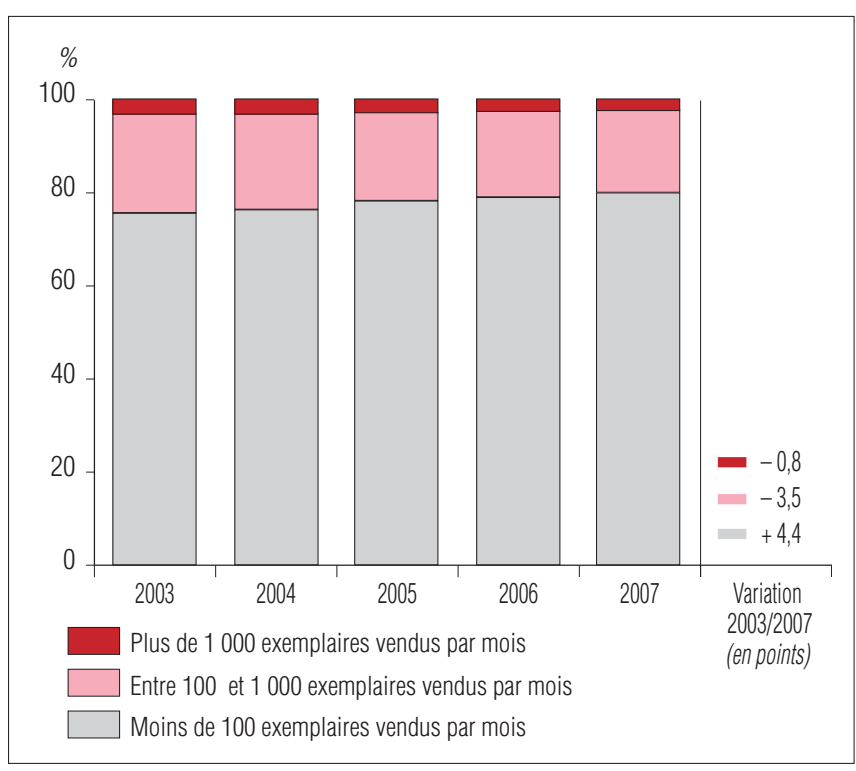

Source : DEPS, Ministère de la Culture et de la Communication, 2011

\section{Graphique 5-Proportion des titres à fortes ventes mensuelles (moyenne annuelle)}

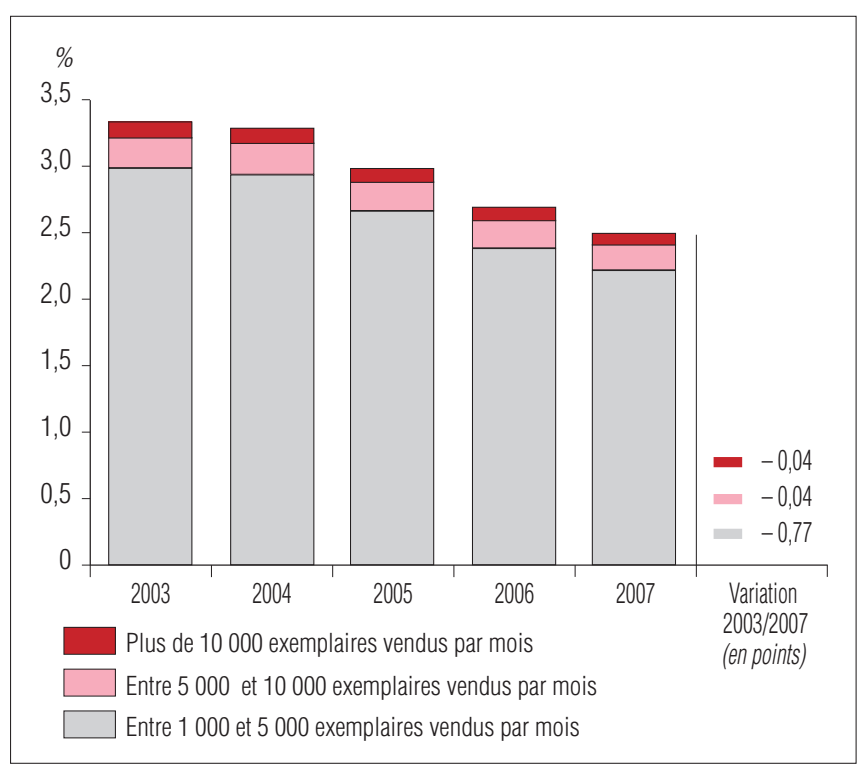

Source : DEPS, Ministère de la Culture et de la Communication, 2011.

\section{Progression du poids des titres confidentiels (entre 5 et 50 exemplaires vendus par mois)}

L'augmentation des ventes de titres à faibles ventes semble profiter essentiellement aux titres dits « confidentiels » (vendus entre 5 et moins de 50 exemplaires par mois). En moyenne, leur part est en effet passée de $34 \%$ à $39 \%$ des titres vendus mensuellement, soit une hausse de 5 points en cinq ans. En revanche, ni la position des titres dits «obscurs» (vendus à moins de 5 exemplaires par mois) ni celle des titres à faible diffusion (vendus entre 50 et moins de 100 exemplaires par mois) ne sont modifiées significativement. La part des titres vendus à moins de 5 exemplaires par mois a augmenté de 2003 à 2005 mais est ensuite revenue en 2007 au niveau observé en début de période, soit $31 \%$. Quant aux titres vendus mensuellement entre 50 et 100 exemplaires, leur part dans le total des titres n'a que très légèrement baissé au cours de la période $(9,7 \%$ des titres en 2007 contre 10,4\% en 2003).

\section{Stabilité du nombre de titres vendant plus de 10000 exemplaires par mois}

Un très faible nombre de livres atteint des niveaux de ventes élevés

En 2007, 2,5\% des titres (2 767 livres) se vendent à plus de 1000 exemplaires par mois, 0,28\% atteint plus de 5000 exemplaires et seulement $0,08 \%$ dépasse 10000 ventes mensuelles. Cette concentration sur quelques titres stars tend à s'éroder légèrement (graphique 5). La concentration des ventes sur un nombre plus réduit de titres recule, entre 2003 et 2007, pour les meilleures ventes (plus de 1000 exemplaires vendus par mois) comme pour les fortes ventes (entre 5000 et 10000 ). Toutefois, lorsque l'on raisonne en nombre de titres, le nombre de titres vendus mensuellement à plus de 5000 exemplaires apparaît en légère croissance sur la période (autour de 300) alors qu'il est globalement stable (autour de 90) pour les titres vendus mensuellement à plus de 10000 exemplaires.

Le précédent constat d'une tendance à la baisse du nombre de titres représentant la moitié des ventes totales ne traduit pas pour autant un accroissement des ventes de quelques best-sellers mais va plutôt de pair avec un nombre stable voire légèrement plus élevé de titres à fortes ventes. Ce résultat est-il validé pour les super-best-sellers?

\section{Accroissement du poids du top $\mathbf{1 0}$}

L'analyse de la part du top 1000 dans les ventes totales, de la part du top 100 dans les ventes totales et de la part du top 10 dans les ventes du top 100 permet de compléter l'analyse de la variété consommée (tableau 5). Selon les deux premiers critères, la polarisation de la demande sur les meilleures ventes est nettement plus élevée en bande dessinée, dans une moindre mesure en littérature qu'en jeunesse. En 2007, le top 1000 réalise près des deux tiers des ventes en bande dessinée (63\%), plus de la moitié en littérature $(55 \%)$ mais seulement $42 \%$ en jeunesse. Les 100 meilleures ventes de livres jeunesse représentent $14 \%$ des ventes, soit près de moitié moins qu'en bande dessinée ou 


\begin{tabular}{|lcccccc|}
\hline & $\mathbf{2 0 0 3}$ & $\mathbf{2 0 0 4}$ & $\mathbf{2 0 0 5}$ & $\mathbf{2 0 0 6}$ & $\mathbf{2 0 0 7}$ & $\begin{array}{c}\text { Variation 2003/2007 } \\
\text { (en points) }\end{array}$ \\
Top 100 & & & & & $-0,75$ \\
Top 1000 & 38,75 & 38,33 & 40,08 & 38,75 & 38,00 & $-0,50$ \\
Top10/top100 & 82,08 & 83,08 & 83,00 & 82,08 & 81,58 & 3,68 \\
\hline
\end{tabular}

Source : DEPS, Ministère de la Culture et de la Communication, 2011.

en littérature (respectivement $26 \%$ et $22 \%$ des ventes). Cependant, la part du top 10 dans le top 100 est légèrement plus élevée en jeunesse qu'en littérature (34\% contre $31 \%$ ). La consommation de livres de jeunesse semble donc plus soumise aux effets de mimétisme sur les super-bestsellers. Les jeunes générations auraient une plus forte tendance à acheter les livres dont tout le monde parle, influencés par de vastes campagnes de promotion ou suivant les avis de leurs proches.

En littérature, quels que soient les tops considérés, on observe une légère tendance au renforcement des best-sellers et plus particulièrement des super-best-sellers. Dans un top 100 dont le poids est passé de $20 \%$ des ventes totales en moyenne sur l'année 2003 à $22 \%$ en 2007, les dix meilleures ventes ont vu leur part encore progresser de 3 points, concentrant $31 \%$ des ventes en 2007 contre $28 \%$ en 2003. Au contraire, pour les bandes dessinées, une tendance nette à la déconcentration des ventes se dégage. Toutefois, ce phénomène semble moins affecter les œuvres à très grand succès que le reste des titres entrants dans les meilleures ventes. Alors que le top 1000 enregistre une baisse de 7 points sur la période (de $70 \%$ à $63 \%$ des ventes totales en moyenne annuelle entre 2003 et 2007), et que le top 100 recule de $31 \%$ à $26 \%$, le poids du top 10 dans ce top 100 reste quasiment stable, autour de $38 \%$. L'érosion des best-sellers affecterait donc peu les superstars de la bande dessinée (Titeuf, Astérix, etc.). Pour la catégorie jeunesse, le poids du top 1000 a également diminué entre 2003 et 2007, même si cette baisse est moins marquée qu'en bande dessinée (de $46 \%$ à $42 \%$ en moyenne annuelle). Ce recul est beaucoup moins marqué pour les cent meilleures ventes, et ne fragilise en rien la position des super-bestsellers. Entre 2003 et 2007, la part du top 10 dans le top 100 a gagné 4 points (de $30 \%$ à $34 \%$ ).

Pour synthétiser l'évolution de la dimension équilibre, le milieu de la distribution se creuse (la part des titres vendus entre 100 et 1000 exemplaires par mois diminue), au bénéfice d'un épaississement de la trâne (les titres vendus entre 5 et 50 exemplaires par mois voient leur importance relative croître de 5 points). En revanche, la part des titres obscurs (moins de 5 ventes mensuelles) reste stable et aurait même tendance à se réduire, sauf en bande dessinée ${ }^{15}$. Toutefois, cet allongement (compte tenu de la forte augmentation du nombre de titres vendus) et cet épaississement de la traîne vont de pair avec un renforcement de la tête de la distribution. La part des titres réalisant la moitié des ventes tend à se réduire sur tous les segments et le poids du top 10 dans le top 100 tend à s'accroître, sauf en bande dessinée.

\section{Disparité}

À variété et équilibre constants, la diversité peut s'accroître si les œuvres consommées présentent une disparité croissante. À l'inverse, un accroissement de la variété et une répartition plus équilibrée des ventes peuvent aller de pair avec une plus grande standardisation des œuvres. Travaillant sur des données quasi exhaustives de ventes, il s'est révélé impossible de conduire une analyse fine de comparaison des contenus des livres. Toutefois, trois types de critères ont été retenus pour rendre compte - partiellement de l'évolution de la disparité :

- le taux de renouvellement des titres dans les tops, qui révèle si les mêmes livres trustent les tops sur de longues périodes ou si, au contraire, le contenu de ces meilleures ventes se renouvelle fréquemment;

- une analyse de la diversité des auteurs, sur la catégorie de la littérature ;

- une étude des éditeurs qui offre un éclairage sur l'évolution de la situation des éditeurs indépendants au cours de la période ainsi que sur leur position sur les ventes en ligne par rapport aux autres circuits. Une position plus affermie des éditeurs indépendants parmi les ventes au cours de la période pourrait être l'indice d'une plus grande diversité.

\section{Une tendance à la baisse des taux de renouvellement des livres dans les tops}

Les titres présents dans les tops, sont-ils identiques d'un mois sur l'autre ou les consommateurs sont-ils de moins en moins fidèles aux mêmes œuvres stars ? Une possible érosion des best-sellers peut tenir à une forte mobilité à l'intérieur du top 100 et non seulement à une réduction de leur poids dans les ventes ou à une réduction de leur nombre. Au niveau des best-sellers, les indicateurs retenus vont dans le sens d'une moindre disparité entre les titres. Pour limiter le risque de se tromper, face à une offre de titres qualifiée souvent de pléthorique, les consommateurs continuent à être fidèles à des œuvres stars dont les temps de passage dans les tops, en règle générale, s'allongent ou, tout du moins, ne sont pas moins courts. Une amélioration de la disparité devrait se traduire par une augmentation des taux de renouvellement des titres au sein du top 100, du pourcentage de titres qui n'étaient pas présents dans le top 100 le mois précédent. Or, on constate une baisse continue du taux de renouvellement en bande dessinée $(-4,5$ points entre 2003 et 2007), un léger déclin en littérature depuis 
2004 ( $-2,5$ points) et une relative stabilité en jeunesse depuis 2005, date à partir de laquelle 39 nouveaux titres, en moyenne annuelle, entrent dans le top 100 mensuel.

Aucune preuve d'un accroissement de la diversité, au sein des 100 meilleures ventes, ne peut donc être apportée de cette manière. Un taux de renouvellement plus faible signifie, au contraire, qu'il est plus difficile pour une nouveauté d'entrer dans la liste des cent meilleures ventes ou qu'un titre présent dans le top 100 a plus de chance de conserver sa position. Les consommateurs concernés ne sont donc pas plus versatiles mais de plus en plus fidèles à un nombre restreint d'œuvres.

\section{Un nombre croissant d'auteurs contrebalancé par un renforcement de l'effet superstar}

On suppose que la disparité augmente également si les titres consommés sont écrits par un nombre d'auteurs croissant et si la demande est moins concentrée sur quelques superstars, laissant alors une part plus importante des ventes aux auteurs jusqu'alors ignorés. Il apparaît clairement que les œuvres littéraires ${ }^{16}$ sont aujourd'hui le fait d'un plus grand nombre d'auteurs : 18200 auteurs en moyenne ont vendu une œuvre littéraire par mois en 2007 contre 14600 en 2003.

L'existence d'un plus grand nombre d'auteurs lus se double-t-elle d'une répartition plus équilibrée des ventes entre eux ? On est tout d'abord frappé par le caractère très inégalitaire des ventes en littérature, encore plus flagrant au niveau des auteurs que des titres. En 2007, $15 \%$ des titres et seulement $5 \%$ des auteurs concentrent $90 \%$ des ventes. $50 \%$ pour les titres mais $64 \%$ des auteurs se partagent $1 \%$ des ventes. De plus, ce phénomène de concentration tend à se renforcer de 2003 à 2007, signe de l'importance des mécanismes d'autorenforcement de la notoriété à l'œuvre dans le secteur du livre.

Les parts de marché des 1000 et des 100 auteurs réalisant les meilleures ventes, après avoir augmenté de 2003 à 2005, reculent depuis pour s'établir à un niveau équivalent à celui observé en début de période. En revanche, un mouvement inverse de concentration s'opère à l'intérieur du top 100 , où les auteurs du top 10 réalisent une part plus importante des ventes : les dix superstars réalisent près de $32 \%$ des ventes du top 100 en 2007 contre $28 \%$ en 2003, soit une progression de 4 points.

\section{Un rôle accru pour les petits éditeurs}

L'édition française présente une structure industrielle d'oligopole à frange concurrentielle. Quelques firmes dominantes se partagent l'essentiel du marché, quelques suiveurs de moindre importance cumulent une bonne partie du reste et une kyrielle de petits éditeurs se partage les miettes. En 2007, Hachette et Editis représentaient à eux seuls, en moyenne chaque mois, $37 \%$ du marché des livres pour la jeunesse et $52 \%$ des ventes de littérature. En bande dessinée, le groupe Media Participation et l'éditeur Glénat cumulaient $51 \%$ des ventes. Une poignée d'éditeurs de moindre importance suit ces leaders mais le dixième éditeur ne réalise pas plus de $1 \%$ des ventes, que ce soit en littérature, en bande dessinée et en livres pour la jeunesse.

Il est d'usage de considérer que, dans les industries culturelles, l'offre émanant des firmes de l'oligopole est qualitativement différente de celle émanant de la frange concurrentielle. Les premières viseraient plus le marché de masse, la culture mainstream ${ }^{17}$, éditant notamment les auteurs à succès, souvent découverts par les petits éditeurs de la frange concurrentielle, les seconds investissant plus souvent des marchés de niche (comme la poésie par exemple). Cette image caricaturale reflète néanmoins une certaine réalité. Lorsque Fred Vargas, auteur de romans policiers à succès, explique qu'elle est restée, depuis ses débuts, fidèle à sa première maison d'édition, Viviane Hamy, elle souligne en creux le caractère inhabituel de sa démarche. «Si les auteurs quittent ceux qui leur ont mis le pied à l'étrier, il ne faut pas se plaindre de la concentration de l'édition et de la disparition des maisons indépendantes ${ }^{18}$.»

L'analyse de l'évolution de la population des éditeurs et de la concentration des ventes par éditeur complète l'analyse de la disparité de la diversité dans l'industrie du livre. Un accroissement du nombre d'éditeurs actifs concourt plutôt à une progression de la disparité, à l'inverse d'une tendance à la concentration des ventes sur un nombre réduit d'éditeurs. En 2007, 1800 éditeurs en moyenne étaient présents chaque mois sur la catégorie littérature $(+29 \%$ par rapport à 2003), 670 en jeunesse $(+52 \%)$ et 457 en bande dessinée $(+41 \%)$. La croissance du nombre d'éditeurs traduit une certaine déconcentration de l'activité éditoriale.

Selon les critères retenus par les autorités européennes de la concurrence, un secteur est considéré comme très concentré si l'indice Herfindhal-Hirschmann ${ }^{19}$ excède la valeur de 2000 , moyennement concentré s'il se situe entre 1000 et 2000 et peu concentré s'il est inférieur à 1000 . De ce point de vue, l'activité éditoriale dans les trois segments doit être considérée, en 2007, comme moyennement concentrée (graphique 6). La baisse la plus importante concerne la bande dessinée, dont l'indice HHI tendait à excéder le niveau de 2000 jusqu' au milieu de l'année 2005 et qui se situe en 2007 à un niveau proche de la littérature autour de 1700 , le degré de concentration de cette dernière n'ayant que très peu baissé sur la période. C'est sur la catégorie des livres pour la jeunesse que la concentration des éditeurs apparaît la plus faible.

Cette baisse de l'indice HHI, toute relative pour la littérature, peut toutefois obéir à des logiques bien différentes. Est-elle due à un renforcement des tout petits éditeurs de

16. Cette analyse n'a pu être menée que pour le domaine de la littérature (voir «Éléments de méthodologie », p. 15).

17. Frédéric MARTEL, Mainstream. Enquête sur cette culture qui plaît à tout le monde, Paris, Flammarion, 2010.

18. Interview dans Télérama, 9 février $2008, \mathrm{n}^{\circ} 3030$.

19. L'indice d'Herfindhal-Hirschmann (HHI) se calcule comme la somme du carré des parts de marché. Il prend une valeur comprise entre 0 (situation où aucun titre n'aurait une part de marché significative) et 10000 (situation où un seul titre s'accaparerait l'ensemble du marché). 


\section{Graphique 6 - Indice de concentration $\mathrm{HHI}$ des ventes (en volume) par éditeur selon la catégorie}

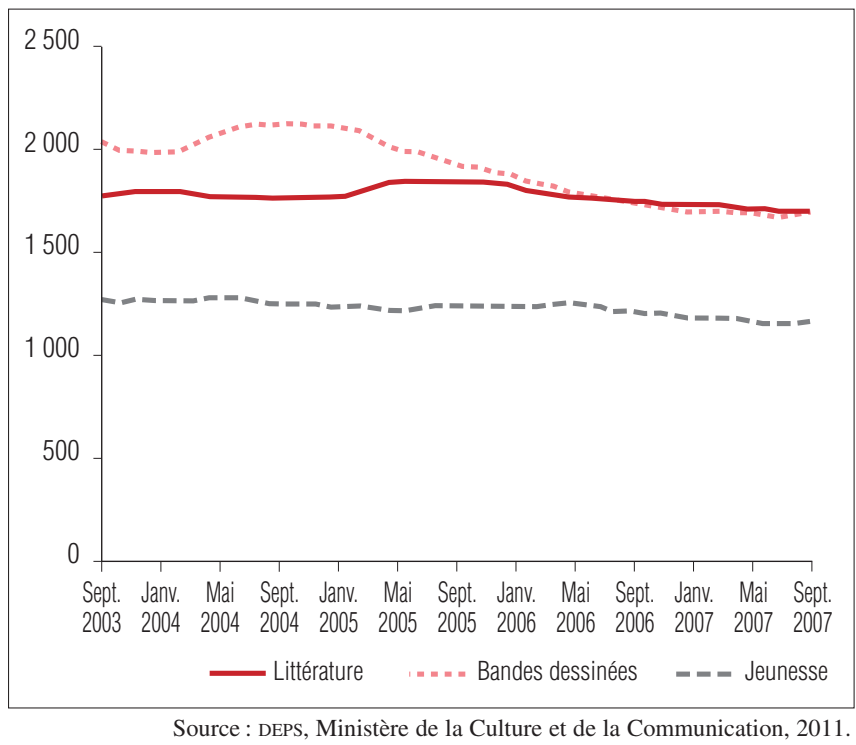

la frange concurrentielle, à une croissance des éditeurs moyens au détriment des éditeurs dominants ou au contraire à une simple redistribution des rôles entre ces éditeurs grands et moyens? L'étude des parts de marché cumulées des quatre premières et dix premières firmes sur chaque segment permet de répondre à ces questions.

Les dynamiques sont assez différentes d'une catégorie à l'autre (tableau 6). En littérature, la hiérarchie a été peu modifiée. La part de marché des quatre groupes les plus importants (Hachette, Editis, Gallimard, Flammarion) s'est légèrement réduite tout comme celle des dix premiers ${ }^{20}$, mais de manière peu significative. Sur la catégorie des livres pour la jeunesse, une érosion de la part de marché des quatre principaux groupes ${ }^{21}$ s'opère au bénéfice presque exclusif des éditeurs les plus importants de la frange concurrentielle ${ }^{22}$. En revanche, en bande dessinée, on observe simultanément une baisse de la part de marché cumulée des quatre premiers ainsi que celle des dix premiers éditeurs, et leur affaiblissement ${ }^{23}$ se fait au profit de l'ensemble de la frange.

L'affaiblissement de l'oligopole s'est effectué au profit d'éditeurs indépendants en bande dessinée (l'Association, Clair de Lune...) alors qu'elle a essentiellement bénéficié aux éditeurs de livres pour la jeunesse, les plus importants appartenant à la frange concurrentielle. En littérature en revanche, les éditeurs de moyenne et de petite taille ne semblent pas être en mesure de contester la position des groupes leaders. Dans ce domaine, le plus concentré parmi ceux étudiés, la part de marché des quatre premières firmes (Hachette, Editis, Gallimard et Flammarion) est restée globalement stable, aux alentours de $76 \%$, sur la période.

\section{Synthèse}

Si le nombre de titres différents achetés a légèrement progressé sur la période, signe d'un accroissement de la variété produite (le nombre de nouvelles références mises sur le marché), on ne peut en conclure pour autant à l'accroissement de la diversité. En matière d'équilibre, le statut des super-best-sellers ne semble pas ou peu s'éroder - le poids du top 10 dans le top 100 des ventes tend même à s'accroître. Ce déplacement de la demande affecte les ventes des titres à moindre succès. Ainsi, le poids des tops 100 et 1000 tend à se réduire dans l'ensemble des ventes. Un double mouvement s'observe donc: un renforcement de la tête de la distribution des ventes et un développement, certes limité, des ventes des titres confidentiels.

L'évolution de la troisième dimension, la disparité, est très contrastée selon les genres. En littérature, si les œuvres sont écrites par un nombre croissant d'auteurs, les ventes tendent à se focaliser sur un nombre de plus en plus restreint d'entre eux. De plus, il existe un faible renouvellement des auteurs à succès, signal d'une faible disparité. Les tops tendent à moins se renouveler, soulignant une persistance toujours plus importante des mêmes titres dans les tops mensuels. Enfin, dans la catégorie de la littérature, l'hégémonie des quatre plus grands groupes (Hachette, Editis, Gallimard et Flammarion) n'apparaît pas contestée. À l'inverse, pour les livres jeunesse et la bande dessinée, le poids des principaux éditeurs dans les ventes totales se réduit continûment au profit des éditeurs de taille plus réduite. Cela peut être retenu comme un indice d'une moindre standardisation dans la mesure où la nature des éditeurs - grands groupes ou petits éditeurs - peut avoir un impact sur les contenus proposés (produits mainstream versus produits de niche).

\section{QUESTIONS CLÉS}

L'étude permet d'apporter des réponses nouvelles ou complémentaires à des questions usuelles traitant de la filière de l'édition : celle relative aux effets de la loi sur le prix unique du livre ${ }^{24}$ ou la question de l'impact de l'internet sur la distribution des ventes de livres en France ${ }^{25}$.

20. Aux quatre leaders invariants sur la période s'ajoutent en 2007 La Martinière-Le Seuil, Albin Michel, Actes Sud, Rivages, Michel Lafon, Libella. 21. Sur la catégorie jeunesse, les quatre principaux groupes d'édition sur l'ensemble de la période sont Hachette, Editis, Gallimard et Bayard. 22. En 2007, la cinquième place était détenue par Média Participations suivi par L'École des loisirs, Flammarion, Albin Michel, Lito et Piccolia. 23. En 2007, ces groupes sont Media Participation, Glénat, Delcourt/Tonkam et Flammarion. Entre la cinquième et dixième place, on trouve les Éditions du Soleil, Hachette, Editis, Panini, Bamboo, Albert René.

24. Voir F. RouET, le Livre. Mutations d'une industrie culturelle, Paris, La Documentation française, 2007 ; H. GAYMARD, Situation du livre..., op. cit. ; J. Perona et J. Pouyet, le Prix unique du livre à l'heure du numérique, Paris, Éditions Rue d’Ulm, coll. « Opuscules du Cepremap », $2010, \mathrm{n}^{\circ}$ 19.

25. P.-J. Benghozi et F. Benhamou, Longue traîne : levier numérique de la diversité culturelle ?, Paris, Ministère de la Culture et de la Communication, DEPS, coll. «Culture prospective», 2008-1 ; D. BounIE, B. EANG et P. WAELBROECK, « Marché internet et réseaux physiques : comparaison des ventes de livres en France », Revue d'économie politique, 2010, n 120, p. 141-162 ; S. PeLTiER et F. MorEAU, "Internet and the 'Long Tail' vs Superstar effect debate: Evidence from the French Book Market", Applied Economic Letters, 2012, 19(8): 711-715. 
Tableau 6 - Répartition du marché entre éditeurs par segment (moyenne des parts de marché mensuelles)

\begin{tabular}{|c|c|c|c|c|c|c|}
\hline & & 2003 & 2004 & 2005 & 2006 & 2007 \\
\hline \multirow[t]{3}{*}{ Littérature } & Parts de marché cumulées des quatre premiers éditeurs $\left(\mathrm{CR}_{4}\right)$ & 77 & 77 & 78 & 76 & 76 \\
\hline & Parts de marché cumulées des six suivants $\left(\mathrm{CR}_{10}-\mathrm{CR}_{4}\right)$ & 12 & 12 & 12 & 13 & 12 \\
\hline & Parts de marché cumulées de tous les autres éditeurs ( $\left.100-\mathrm{CR}_{10}\right)$ & 11 & 11 & 10 & 11 & 12 \\
\hline \multirow[t]{3}{*}{ Bandes dessinées } & Parts de marché cumulées des quatre premiers éditeurs $\left(\mathrm{CR}_{4}\right)$ & 76 & 76 & 73 & 70 & 69 \\
\hline & Parts de marché cumulées des six suivants $\left(\mathrm{CR}_{10}-\mathrm{CR}_{4}\right)$ & 18 & 18 & 20 & 23 & 22 \\
\hline & Parts de marché cumulées de tous les autres éditeurs $\left(100-\mathrm{CR}_{10}\right)$ & 6 & 6 & 7 & 7 & 9 \\
\hline \multirow[t]{3}{*}{ Jeunesse } & Parts de marché cumulées des quatre premiers éditeurs $\left(\mathrm{CR}_{4}\right)$ & 66 & 65 & 65 & 64 & 62 \\
\hline & Parts de marché cumulées des six suivants $\left(\mathrm{CR}_{10}-\mathrm{CR}_{4}\right)$ & 22 & 22 & 23 & 24 & 25 \\
\hline & Parts de marché cumulées de tous les autres éditeurs (100 - $\left.\mathrm{CR}_{10}\right)$ & 12 & 13 & 12 & 12 & 13 \\
\hline
\end{tabular}

Source : DEPS, Ministère de la Culture et de la Communication, 2011.

\section{La loi sur le prix unique favorise-t-elle la diversité culturelle?}

Contrairement à la musique où l'absence de régulation par un système de prix fixe a certainement contribué, avec la crise des ventes du début des années 1980, à l'étiolement du réseau des disquaires indépendants avant même l'apparition des nouvelles technologies, force est de constater que la loi du 10 août 1981 a su garantir un réseau dense et diversifié de librairies. En effet, les grande surfaces alimentaires (GSA) et les grandes surfaces spécialisées (GSS) ne concentrent, fin 2007, que $55 \%$ des livres vendus contre $85 \%$ des ventes de musique enregistrée (CD). Grandes et petites librairies continuent de représenter le circuit de vente d'œuvres littéraires, de livres pour la jeunesse et de bandes dessinées, en volume, le plus important (41\% contre $27 \%$ pour les grandes surfaces alimentaires comme pour les grandes surfaces spécialisées). L'importance de ce réseau de diffusion permet que la diversité produite, et plus largement offerte, « le soit en tous points du territoire et au même prix, éliminant les coûts de la recherche du meilleur prix et minimisant les coûts de déplacement indispensables que l'on rencontre dans des pays moins irrigués en matière de vente au détail ${ }^{26} »$. Dans les librairies, la variété de titres consommés a continué de progresser même si elles se positionnent en termes de nombre de titres différents consommés derrière les grandes surfaces spécialisées et l'internet.

\section{Les librairies : moins de titres qu'en grandes surfaces spécialisées ou que sur l'internet...}

Le nombre de références différentes vendues est en effet plus important dans les grandes surfaces spécialisées (tableau 7), réputées être mieux achalandées que les autres circuits. En 2007, par exemple, avec près de 84000 titres différents vendus chaque mois, la variété consommée est 2,5 fois plus élevée dans ce circuit que dans la grande distribution alimentaire, 2,3 fois plus importante que dans les petites librairies et seulement 1,4 et 1,2 fois plus forte que dans les grandes librairies et l'internet. La GSA confirme son statut de pourvoyeur d'une variété limitée, plutôt tournée vers les livres à fort tirages. Exception faite de l'internet, les grandes librairies représentent le circuit qui a enregistré la plus forte progression (+ $45 \%$ entre 2003 et $2007^{27}$ ).

\section{... mais une place plus importante accordée aux titres confidentiels}

L'analyse de l'équilibre comparé de la distribution des ventes dans les petites et les grandes librairies d'une part, et dans les autres circuits d'autre part, est potentiellement biaisée par le nombre de références disponibles dans chacun des circuits et leur volume de ventes. Raisonner sur la part de marché obtenue par une proportion donnée des titres apparaît le plus pertinent.

Tableau 7 - Nombre moyen de titres différents vendus mensuellement par an par circuit (2003-2007)

\begin{tabular}{|c|c|c|c|c|c|c|}
\hline & 2003 & 2004 & 2005 & 2006 & 2007 & $\begin{array}{c}\text { Évolution 2003-2007 } \\
\text { (en \%) }\end{array}$ \\
\hline Grande librairie & 43130 & 49090 & 52670 & 58310 & 62530 & 45 \\
\hline Petite libraire & 29300 & 31710 & 32310 & 32470 & 36110 & 23 \\
\hline Grande surface spécialisée & 73110 & 76380 & 78590 & 82630 & 83670 & 14 \\
\hline Grande surface alimentaire & 26980 & 29860 & 30600 & 33020 & 33540 & 24 \\
\hline Internet & 28640 & 40660 & 52720 & 60290 & 69760 & 143 \\
\hline
\end{tabular}

26. F. Benhamou, S. Peltier, «La diversité culturelle et le livre», dans H. Gaymard, Situation du livre..., op. cit., Annexe 7, p. 344-348. www.culture.gouv.fr/culture/actualites/rapports/rapport_gaymard.pdf

27. En ce qui concerne les grandes librairies, mais aussi les petites, une partie de cette hausse pourrait s'expliquer par la méthode d'extrapolation des données utilisée par GfK, et plus précisément par l'intégration dans le panel, au fur et à mesure des années, d'un plus grand nombre de librairies présentant des spécificités dans les références offertes par rapport à celles déjà recensées. 
La part de marché minimale des $1 \%$ des titres se vendant le mieux tend à être moins élevée dans les petites librairies qu'en GSA, mais plus importante que dans les grandes librairies et surtout qu'en GSS. La concentration des ventes sur un nombre réduit de titres existe donc bien également dans les petites et les grandes librairies. Toutefois, cette tendance à la domination des tops plus marquée qu'en GSS est pondérée, pour les librairies, par un meilleur sort réservé aux titres à faible diffusion. En effet, la part de marché du titre médian - c'est-à-dire du titre séparant la population en deux sous-populations égales - est plus élevée dans les petites librairies, et dans une moindre mesure dans les grandes librairies, qu'elle ne l'est en GSA et en GSS.

Les petites et grandes librairies présentent donc bien une spécificité : allier les ventes des best-sellers (ces ventes dont les libraires disent qu' «elles se font toutes seules ») à celles de titres beaucoup plus confidentiels qui parviennent à s'octroyer en librairie une part de marché plus importante que celle obtenue en GSs. Moins noyés dans une offre de plus en plus pléthorique, ces titres émergent alors plus facilement.

De même, la substitution d'une concurrence par le prix par une concurrence par la qualité semble avoir joué un rôle dans la défense de la diversité. Quelle que soit la catégorie considérée, ce sont les petites et grandes librairies qui, parmi les circuits traditionnels, offrent systématiquement une place plus importante aux petites maisons d'édition.

De plus, en littérature, le poids des dix auteurs les mieux vendus est moins important dans les grandes ou petites librairies que dans les autres circuits. Les ventes des dix premiers auteurs représentaient, en 2007, $33 \%$ des ventes du top 100 en GSS contre respectivement $31 \%$ et $27 \%$ pour les petites et grandes librairies. Le rôle de conseil des libraires apparaît donc essentiel pour favoriser l'achat de premières œuvres et d'auteurs peu ou moins connus.

La limite de cet exercice de comparaison tient à l'impossibilité de mener une analyse contrefactuelle, c'est-àdire de comparer ces évolutions avec celles qui auraient eu lieu dans un autre contexte législatif. Pour autant, l'analyse permet d'avancer que la loi aura permis de protéger quelque peu la diversité, au sens du pluralisme des éditeurs, des lieux de diffusion et de la défense des livres les plus difficiles.

\section{Les ventes en ligne représentent-elles un levier de la diversité culturelle?}

Les ventes sur l'internet de livres en littérature, bandes dessinées et jeunesse (hors livres numériques), bien qu'en très forte progression, ne représentent encore qu'une part inférieure à $4 \%$ en 2007 (exactement 3,6\% en volume et $3,7 \%$ en valeur). Même si le rôle de l'internet est encore modeste, peut-on anticiper qu'un développement des ventes en ligne aura un impact significatif sur la diversité culturelle?

\section{L'internet :}

\section{un effet positif sur la variété consommée}

Tout d'abord, l'internet joue un rôle important dans l'accroissement de la variété consommée sur la période (69 760 titres différents vendus mensuellement en moyenne en 2007, soit 41000 titres de plus qu'en 2003). L'internet représente désormais le deuxième circuit de distribution selon ce critère derrière les GSS (83 670 titres différents vendus mensuellement en 2007 mais seulement 10500 titres de plus qu'en 2003). La variété consommée sur l'internet représente ainsi plus de $80 \%$ de la variété consommée en $\mathrm{GSS}^{28}$. Certes, l'ampleur de la hausse de la variété consommée sur l'internet s'explique pour partie par la croissance des ventes en ligne ${ }^{29}$ et par l'intégration dans le panel de nouveaux sites de ventes en ligne au cours de la période (Fnac.com, Alapage). Toutefois, depuis fin 2004, date de l'entrée de Fnac.com dans le panel, la hausse reste soutenue.

Dans quelle mesure l'internet a-t-il contribué à la croissance de la variété consommée au niveau global ? S'il est difficile de répondre à cette question avec précision, quelques pistes de réflexion méritent d'être dessinées. Au niveau agrégé, le nombre de références vendues a augmenté de 25800 entre 2003 et 2007. Dans le même temps, la progression en GSS, le circuit le mieux achalandé, n'a été que de 10600 références. Il est donc probable que les quelque 15000 nouvelles références vendues qui ne proviennent pas de la GSS soient liées - pour partie tout au moins - à l'essor des ventes de livres sur l'internet. Les références vendues y ont en effet augmenté de plus de 40100 unités. Une bonne partie constitue sans nul doute un sous-ensemble des références vendues en GSS, mais certainement pas la totalité ${ }^{30}$. D'autant que si l'accroissement sur la période du nombre de vendeurs en ligne a manifestement eu pour conséquence une augmentation du nombre de références différentes vendues sur l'internet, à l'inverse il est souvent reproché aux GSS d'avoir dans le même temps resserré leurs assortiments. Par ailleurs, si en 2003, $14 \%$

\footnotetext{
28. D'autant que si un consommateur peut avoir accès à l'ensemble de l'offre en ligne quel que soit son lieu de résidence, il n'en est pas de même pour l'offre physique dans les circuits traditionnels qui se limite aux magasins proches de chez lui.

29. Sur l'internet, l'augmentation de la variété consommée a également été favorisée par l'engouement qu'a connu ce canal de vente sur la période. Selon le panel consommateurs TNS/Sofres, si seule 5,7\% de la population de 15 ans et plus avait déjà acheté un livre sur l'internet en 2003, ce taux était monté à $16 \%$ en 2007.

30. On pourrait faire l'hypothèse que la forte augmentation des références différentes vendues sur l'internet serait liée uniquement à une forte hausse des ventes de titres de littérature en vo, mais il apparaît qu'il n'en est rien. Alors que l'accroissement des références vendues mensuellement sur l'internet a été en moyenne de 18900 entre 2003 et 2007, le nombre de titres en vo sur ce même circuit a augmenté de seulement 1600 , soit à peine $9 \%$.
} 
seulement des titres différents vendus n'avaient pas été achetés au moins une fois en $\mathrm{GSS}^{31}$; en 2007, cette part s'élève à $26 \%$.

\section{Un effet ambigu sur la concentration des ventes}

Le développement des ventes en ligne a un effet contrasté sur l'équilibre des ventes. L'étude montre une répartition plus égalitaire des ventes sur l'internet que sur les autres circuits de distribution (moins prononcée toutefois sur la catégorie jeunesse). Ainsi, la part des titres représentant $50 \%$ des ventes est plus de deux fois plus élevée sur l'internet qu'en GSS ( $7 \%$ contre $3 \%$ en bande dessinée et $4 \%$ contre $1,5 \%$ en littérature fin 2007), sauf en jeunesse où elle est très proche (autour de $5 \%$ fin 2007). De même, en moyenne sur l'année 2007, la part de marché minimale des $1 \%$ des titres se vendant le mieux était plus élevée en GSS qu'en ligne (hormis pour la catégorie jeunesse): les meilleures ventes occupent donc une place moins prépondérante sur l'internet. En revanche, la part de marché qui séparait la population des titres en deux était plus élevée sur l'internet qu'en Gss qu'en ligne, illustrant le fait que les titres à faible vente y sont plus favorisés ${ }^{32}$.

Conformément à la thèse d'Anderson ${ }^{33}$, il apparaît que sur l'internet les meilleures ventes se partagent entre plus

\section{Tableau 8-Part des auteurs et part des ventes pour la littérature en GSS}

\begin{tabular}{|lrrrrr|}
\hline $\begin{array}{l}\text { Part des auteurs } \\
\text { cumulant... }\end{array}$ & $\mathbf{2 0 0 3}$ & $\mathbf{2 0 0 4}$ & $\mathbf{2 0 0 5}$ & $\mathbf{2 0 0 6}$ & $\mathbf{2 0 0 7}$ \\
$50 \%$ des ventes & 1,4 & 1,3 & 1,3 & 1,2 & 1,3 \\
$40 \%$ des ventes & 4,7 & 4,6 & 4,6 & 4,6 & 4,6 \\
$9 \%$ des ventes & 36,5 & 36,1 & 36,2 & 36,2 & 36,1 \\
$1 \%$ des ventes & 57,3 & 58,0 & 58,0 & 58,0 & 58,0 \\
Total & $\mathbf{1 0 0}$ & $\mathbf{1 0 0}$ & $\mathbf{1 0 0}$ & $\mathbf{1 0 0}$ & $\mathbf{1 0 0}$ \\
\end{tabular}

Source : DEPS, Ministère de la Culture et de la Communication, 2011.

\section{Tableau 9 - Part des auteurs et part des ventes} pour la littérature sur l'internet

\begin{tabular}{|lrrrrr|}
\hline $\begin{array}{l}\text { Part des auteurs } \\
\text { cumulant... }\end{array}$ & $\mathbf{2 0 0 3}$ & $\mathbf{2 0 0 4}$ & $\mathbf{2 0 0 5}$ & $\mathbf{2 0 0 6}$ & $\mathbf{2 0 0 7}$ \\
$50 \%$ des ventes & 3,3 & 2,8 & 2,2 & 2,2 & 2,0 \\
$40 \%$ des ventes & 12,7 & 11,1 & 8,8 & 8,5 & 7,7 \\
$9 \%$ des ventes & 63,8 & 61,2 & 72,0 & 71,2 & 71,4 \\
$1 \%$ des ventes & 20,2 & 24,9 & 17,0 & 18,1 & 18,9 \\
Total & $\mathbf{1 0 0}$ & $\mathbf{1 0 0}$ & $\mathbf{1 0 0}$ & $\mathbf{1 0 0}$ & $\mathbf{1 0 0}$ \\
\hline
\end{tabular}

Source : DEPS, Ministère de la Culture et de la Communication, 2011

\section{Graphique 7 - Part du top 10 dans le top 100} par circuit (littérature)

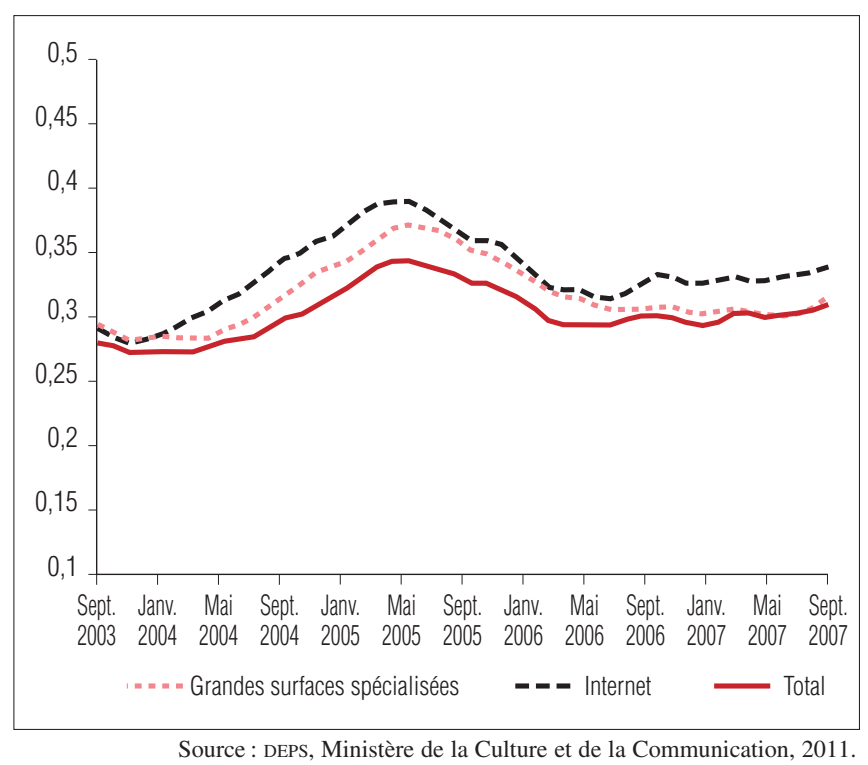

de titres et que les ventes de la queue de la distribution y sont proportionnellement plus importantes. Parallèlement, cette analyse suggère une augmentation de la concentration, avec une part de plus en plus réduite de titres qui s'accapare $50 \%$ des ventes totales. Là encore, ce résultat semble cohérent avec les analyses empiriques de la longue traîne tendant à montrer un renforcement des best-sellers en parallèle avec le développement d'une longue traîne. L'analyse des tops, et donc des super-best-sellers, confirme ces résultats. La part du top 10 dans le top 100 est plus élevée sur l'internet que dans les autres circuits en littérature et en jeunesse et au même niveau, voire inférieure, pour la bande dessinée. De plus, sur les différents segments, le poids des super-best-sellers tend plutôt à se renforcer sur l'internet par rapport aux autres circuits (graphique 7). Tout se passe comme si, avec la démocratisation des ventes en ligne, des achats par des lecteurs épisodiques se matérialisent maintenant en ligne, au détriment, peut-être, de la GSA.

\section{Des ventes en ligne plus favorables aux auteurs méconnus mais aussi aux superstars}

Pour la troisième dimension, la disparité, aucune spécificité des ventes en ligne par rapport aux ventes physiques ne se dégage pour les meilleures ventes. Les bestsellers vendus sur l'internet ne sont pas très différents de ceux achetés en grandes surfaces spécialisées et en librairies. Le taux de renouvellement des titres à l'intérieur des tops n'est pas plus élevé que dans les autres circuits. Les ventes sur l'internet se caractérisent néanmoins par un effet star (concentration des ventes sur un petit nombre d'auteurs à succès) moins marqué (tableaux 8 et 9 ) : $2 \%$ des

31. En moyenne, chaque mois, 85100 titres différents ont été achetés au moins une fois en 2003, mais sur le seul circuit GSs ce chiffre n'atteignait que 73 100, soit un taux de couverture de $86 \%$. Autrement dit, en moyenne, sur chaque mois de 2003, $14 \%$ des titres vendus au moins une fois n'avaient enregistré aucune vente en GSS.

32. Cette meilleure position est toute relative. La part de l'internet sur le marché du livre est si réduite, que pour un titre donné, la plus faible part de marché réalisée en GSS qu'en ligne ne l'empêche pas d'avoir un volume de vente plus élevé sur le premier circuit que sur le second.

33. C. ANDERSON, The Long Tail, op. cit. 
auteurs réalisent $50 \%$ des ventes en ligne contre 1,26\% des auteurs en GSS et $1,14 \%$ tous circuits confondus. Il faut près de $10 \%$ des auteurs pour atteindre $90 \%$ des ventes sur l'internet, un chiffre près de deux fois plus élevé qu'en GSS $(6 \%)$ et que pour les ventes tous circuits confondus $(5 \%)$. En revanche, la situation des auteurs les moins vendus est nettement meilleure pour les ventes en ligne. Si environ deux tiers des auteurs se partagent $1 \%$ des ventes totales, cette part tombe à $40 \%$ en GSS et à moins de $20 \%$ sur l'internet.

Les résultats sont radicalement différents lorsqu'on s'intéresse aux parts de marché des superstars, ces quelques écrivains à très forte notoriété. Les évolutions de la part du top 10 dans le top 100 auteurs, bien que très sensibles à la sortie d'un ou plusieurs titres à forte notoriété (comme l'édition poche du Da Vinci Code en mai 2005), révèlent globalement un mouvement de concentration sur la short head (les fortes ventes des superstars) sur tous les circuits. Ce renforcement du star-system apparaît plus important sur l'internet. Sur ce circuit, si les dix premiers auteurs de littérature réalisaient, en moyenne, $27 \%$ des ventes mensuelles du top 100 en 2003 , leur part atteint près de $32 \%$ en 2007.

Alors que l'effet «star» est plus net pour les ventes physiques que sur l'internet au niveau du top 100 et du top 1000 , les superstars sont plus prépondérantes sur l'internet qu'en grandes librairies et petites librairies. Ce résultat témoigne de l'importance du rôle des libraires pour mettre en avant tel ou tel titre afin de favoriser l'émergence de nouveaux talents ou le succès d'auteurs peu connus. Si l'internet favorise également ces auteurs, les stratégies des sites consistant à afficher les listes des dix meilleurs auteurs et les titres en page d'accueil conduiraient à une concentration des ventes encore plus forte sur ces produits. L'effet dit «page d'accueil» (home page) viendrait contrebalancer l'effet «longue traîne».

Enfin, alors que la part de marchés des quatre premiers éditeurs $\left(\mathrm{CR}_{4}\right)$ est plus faible en moyenne d'environ 5 points sur l'internet par rapport à la GSS pour la littérature et les livres pour la jeunesse, en fin de période la part de marché de Hachette et Editis est plus faible de 7 points pour la littérature et de 10 points pour les livres pour la jeunesse sur l'internet par rapport aux grandes surfaces culturelles. Un phénomène identique se produit pour la catégorie de la bande dessinée, bien que Hachette et Editis n'y soient pas dominants. Leurs parts de marché sur l'internet sont plus faibles que dans les autres circuits, même si la différence est de moindre ampleur. Tout se passe comme s'il était plus facile aux éditeurs n'appartenant pas au duopole d'exister sur le circuit le moins contrôlable par les moyens traditionnels du marketing. Il leur est aussi plus facile d'être présents sur le circuit où le contrôle de puissantes sociétés de diffusion-distribution ne se révèle pas un avantage concurrentiel essentiel, comme cela peut être le cas pour les circuits traditionnels.
La structure particulière de la distribution des ventes entre les titres sur l'internet est cohérente avec l'hypothèse de la longue traîne de Chris Anderson ${ }^{34}$, selon laquelle l'internet représente une chance pour les livres à rotation lente dont les ventes confidentielles rebutent les libraires en quête de place pour les nouveaux titres. Or, sur l'internet, l'absence de contrainte d'exposition doit permettre de vendre ces livres sur la durée et donc de leur laisser le temps de trouver leur lectorat. La longue traîne comprend donc l'ensemble des titres pour lesquels un public suffisamment important existe pour que les produits s'avèrent rentables, mais qui est trop épars pour que les commerces de détail soient en mesure de les proposer durablement. Ainsi, un plus grand nombre de titres est accessible et la demande peut donc potentiellement se détourner de la tête de distribution (steep tail) (les best-sellers) pour se diriger vers la long tail. Les modes de promotion décentralisée autorisés par l'internet (forums, blogs, conseils d'achats sur les sites des marchands en ligne, etc.) peuvent contribuer au transfert de demande. Grâce à l'internet, non seulement des titres non proposés dans les librairies physiques sont accessibles, mais en plus les consommateurs peuvent en avoir connaissance et recueillir des avis d'internautes les ayant déjà lus. L'augmentation de la part des titres à faible tirage dans les ventes totales, au détriment des titres à plus fortes ventes, observée sur le circuit des ventes en ligne semble donc cohérente avec cette analyse. Toutefois, on note également la résistance des super-best-sellers. Cette observation est également conforme avec de précédents travaux sur la longue traîne qui montraient que la réduction du nombre d'œuvres superstars pouvait pour partie avoir pour conséquence un renforcement de la demande sur celles restantes ${ }^{35}$.

34. C. Anderson, The Long Tail, op. cit.

35. E. Brynjolfsson, Y. J. Hu et D. Simester, "Goodbye Pareto Principle, Hello Long Tail: The Effect of Search Costs on the Concentration of Product Sales", Management Science, 2011, à paraître ; F. OBERHOLZER-GEE et A. Elberse, "Superstars and Underdogs: An Examination of the Long Tail Phenomenon in Video Sales", Working paper, n 07.015, Harvard Business School. 


\section{Les données}

Cette étude prend pour base les données sur les ventes physiques de livres en France collectées par l'institut GfK sur une base mensuelle couvrant une période de cinq années (de janvier 2003 à décembre 2007). L'analyse s'est focalisée sur les ouvrages qui appartiennent aux trois segments suivants : la littérature générale ; les livres pour la jeunesse (hors coloriage, stickers et autocollants); les bandes dessinées.

L'Institut GfK recueille les données brutes de ventes hebdomadaires en sortie de caisse dans environ 2300 points de vente en 2007. Le nombre de magasins audités a augmenté chaque année sensiblement ${ }^{1}$, les données en sortie de caisse recueillies étant extrapolées de façon à représenter les ventes au niveau national. Les coefficients de pondération sont ajustés au fur et à mesure des intégrations de nouveaux points de vente. Selon GfK, les données extrapolées représentent la quasi-totalité des ventes physiques de livres en France (en dehors des clubs France Loisirs, de la vente par correspondance (VPC), des ventes des sites étrangers et des ventes aux collectivités). Les données s'entendent hors ventes directes des éditeurs, ventes à terme (collectivités et entreprises), soldeurs, ventes de livres d'occasion, ventes de livres dans les boutiques des musées et dans les stations-service. GfK n'a pu communiquer les informations sur le circuit des clubs pour des raisons de confidentialité car seul France Loisirs, qui a acquis $50 \%$ du Grand livre du mois en 2004 et la moitié restante fin 2008, est aujourd'hui présent sur ces circuits de vente.

Les ventes ont été obtenues pour cinq différents réseaux de distribution : les grandes librairies comprenant les librairies dont le chiffre d'affaires annuel est supérieur à $750000 €$; les petites librairies dont le chiffre d'affaires annuel est inférieur ou égal à $750000 €(y$ compris les petits Relay), qui incluent les petites librairies généralistes et la majeure partie des librairies spécialisées (jeunesse, BD, etc. ${ }^{2}$ ) ; les grandes surfaces spécialisées (Gss culturelles qui regroupent les enseignes de la Fnac, Virgin, Cultura et les Espaces culturels Leclerc ${ }^{3}$ ); les enseignes des grandes surfaces alimentaires (GSA) hyper et supermarchés ${ }^{4}$; l'internet, circuit pour lequel, selon GfK, tous les distributeurs en ligne sont audités ${ }^{5}$, pour leurs ventes de livres neufs ${ }^{6}$. Les ventes de livres numériques (e-books) ne sont pas disponibles mais elles demeurent pour l'instant marginales. Le livre numérique est estimé aujourd'hui en France à moins de $0,1 \%$ du marché de l'édition.

Les données collectées par GfK correspondent à des références commerciales, appelées EAN. Pour chaque EAN, GfK nous a communiqué les 23 variables suivantes communes à l'ensemble de la période: mois d'extraction; semaine de première vente; auteur; titre; éditeur ; groupe éditeur ; format: poche, non-poche et beaux-livres ${ }^{7}$; œuvre française ou étrangère (pour la littérature uniquement); données de ventes (quantités et chiffre d'affaires) par canal de ventes: grandes librairies, petites librairies, GSs culturelles, GSA, internet, total (tous circuits confondus) ; trois niveaux de segmentation : 3 segments de niveau 1 (littérature générale, livres pour la jeunesse, bandes dessinées), 15 segments de niveau 2 et 69 segments de niveau $3^{8}$.

Sur la période analysée, on dénombre plus de 253449 EAN différents composés de 150769 livres de littérature générale (59,5\% de l'ensemble des EAN), 68625 livres pour la jeunesse (27,1\%) et 33672 bandes dessinées (13,3\%). Les références correspondent pour plus des deux tiers à des livres qui ne sont pas édités en format poche (172607 références, 68,1\% pour être exact), moins d'un tiers sont des poches (78966 références, soit $31,2 \%$ ) et moins de $1 \%$ sont répertoriés comme des beaux-livres (1493 références, soit $\left.0,6 \%{ }^{9}\right)$.

Un même contenu pouvant apparaître sous plusieurs EAN différents, notamment s'il est simultanément commercialisé en non-poche et en poche, le risque de surestimer le nombre de titres différents vendus pourrait exister. Mais la phase de lancement du cycle de vie du poche signe généralement l'entrée dans la phase de déclin de la version en grand format. En effet, " de nombreux libraires ont désormais pour habitude de retourner le grand format lorsque le titre sort en poche, ce qui tend à raccourcir sa durée de vie et à augmenter la rotation des titres ${ }^{10} "$. À une date donnée, c'est donc le plus souvent le nouveau livre d'un auteur qui est disponible en grand format et ses livres précédents qui font l'objet de promotion en format poche. Un même livre peut être commercialisé sous plusieurS EAN dans d'autres situations qui demeurent marginales : lorsqu'un titre est disponible en version originale et traduit ou pour les œuvres classiques tombées dans le domaine public qui peuvent faire l'objet de plusieurs éditions. La question de l'existence de plusieurs EAN pour un même livre est donc relativement rare dans l'édition et n'a par conséquent pas fait l'objet de traitement particulier. En revanche, les données fournies par GfK sont brutes et donc logiquement n'ont pas pu être exploitées directement. Le principal problème posé par ces données non retraitées concerne le champ «auteur ». En effet, les mesures de la diversité utilisant l'auteur comme unité d'analyse supposent de pouvoir réunir les EAN correspondant à un seul et même auteur. Or le champ «auteur » de la base «brute » de GfK n'est pas toujours renseigné de manière homogène. Lorsque l'auteur est unique, ses nom et prénom ne sont pas toujours inscrits de manière identique. Un retraitement a donc été nécessaire ${ }^{11}$. Par ailleurs, pour les segments «jeunesse » et «bandes dessinées ", l'écriture à plusieurs auteurs (scénariste et dessinateur notamment) est plus la règle que l'exception. Or, le champ «auteur » comprend parfois un seul des deux auteurs, parfois les deux. Ce problème est loin d'être trivial car, notamment

1. Le nombre de points de vente était de 1800 en 2004 et 2005, 2100 début 2006, et plus récemment 2800 en 2008 et 3500 en 2009.

2. Sur l'économie des librairies spécialisées, voir Situation économique de la librairie indépendante, rapport des enquêtes quantitatives, oEL/lpsos, 2007, notamment p. 632. Sur.

3. Pour des raisons de confidentialité, il ne nous a pas été possible de disposer des données de ventes uniquement pour les grands magasins (essentiellement le Bon Marché et les Galeries Lafayette) et les grands kiosques de gare (Relay). Ils ont été agrégés avec les Gss culturelles.

4. Les ventes des grandes surfaces spécialisées non-livres (comme Darty, Leroy Merlin) et des spécialistes du jeu/jouet (comme Toys'R'Us, la Grande Récrée, etc.) n'ont pu être agrégées dans une catégorie "autre " par souci de confidentialité. En raison du poids relativement faible, pour ces enseignes, du segment livre dans leur chiffre d'affaires, cette catégorie de magasins a été intégrée à la GSA.

5. Pour les plus grands: Amazon depuis juillet 2003, Alapage depuis mars 2004, fnac.com depuis septembre 2004, chapitre.com depuis décembre 2007.

6 . Les ventes liées à l'acquisition d'ouvrages via des tiers en passant par un site internet (ce que l'on dénomme souvent par le terme marketplace) ne sont pas considérées.

7. Les livres en format semi-poche, compte tenu de la faiblesse relative de leur effectif, sont classés en non-poche.

8. Nous avons toutefois été contraints de réduire quelque peu le degré de détail de notre analyse. Les distinctions poche/non-poche et des différentes catégories d'ouvrages à l'intérieur du segment littérature n'apparaissent pas suffisamment marquantes pour justifier de maintenir ces distinctions.

9. Il est à noter que la catégorie et le format ne sont pas spécifiés pour 383 EAN (soit $0,2 \%)$.

10. E. MARTI, les Enjeux du livre au format de poche, Paris, Ministère de la Culture et de la Communication, DEPS, coll. «Culture études ", $2008-4$.

11. Sachant que la base contient 60 fichiers mensuels comprenant chacun plus de 80000 EAN, la correction du champ " auteur » s'est vite révélée être un problème complexe. La correction de la base a nécessité la mise en place d'une méthode semi-automatique. Les principes de cette méthode sont les suivants : (i) compilation des 60 fichiers mensuels pour identifier les auteurs potentiellement différents (soit 65816 lignes), (ii) mise en place d'un programme pour identifier les cas d'auteurs sans prénom (de type AUSTER) (3199 cas) et les cas d'auteurs dont le prénom est seulement identifié par la première lettre (de type AUSTER $P$.) et qui possèdent le même nom qu'une autre ligne de la base (2074 cas); (iii) pour chacun de ces 5273 cas ainsi répertoriés, une correction manuelle auteur par auteur a été réalisée. Le même problème a été rencontré pour le champ «éditeur », il a été traité de façon identique. 
dans la bande dessinée, les coauteurs peuvent changer d'une série à l'autre, voire d'un épisode à l'autre. Aussi, nous avons choisi de ne pas effectuer de mesures de la diversité au niveau des auteurs pour les deux segments des livres pour la jeunesse et de la bande dessinée.

II convient également de noter que la méthode adoptée par GfK pour compenser la non-exhaustivité de la collecte des données « sortie de caisse » sur certains circuits, notamment ceux des petites et grandes librairies, permet d'extrapoler les quantités totales vendues, mais pas le nombre de titres différents vendus. Ainsi, les petites et grandes librairies intégrées au fur et à mesure dans le panel présentaient probablement des spécificités régionales voire locales influant sur les titres qui y étaient proposés. Une hausse du nombre de titres différents vendus dans les circuits où la collecte des données n'était pas initialement exhaustive peut ainsi partiellement refléter un simple biais statistique. Cette limite - qui peut minorer l'ampleur des phénomènes décrits mais pas remettre en cause leur existence - est rappelée lorsque cela s'avère nécessaire.

\section{Éléments de méthodologie*}

Les trois dimensions de la diversité peuvent s'interpréter de la manière suivante : la variété correspond au nombre total de références différentes répertoriées, l'équilibre représente la répartition du nombre total d'exemplaires vendus entre ces différentes références, et la disparité étudie la nature et le degré de différenciation entre les références. La diversité est croissante avec ces trois dimensions. Plus la variété, l'équilibre et la disparité seront forts, plus la diversité sera élevée ; toutefois, chaque dimension représente une condition nécessaire mais individuellement insuffisante pour évaluer le niveau de diversité (figure 1).

Selon la première dimension, la variété, la diversité s'accroît avec le nombre de références produites et consommées. Pour la deuxième dimension, l'équilibre, la diversité augmente avec l'uniformité de la distribution des ventes sur les différentes références. Sous l'angle de la troisième dimension, la disparité, la diversité est d'autant plus grande que les références reflètent de fortes spécificités les différenciant nettement les unes des autres. Ce concept de disparité semble être le plus difficile à mettre en œuvre et il faut indubitablement faire preuve de pragmatisme. Pour évaluer la disparité entre les œuvres, une analyse de leurs contenus peut apparaître éminemment subjective et également, ou surtout, très difficile à appliquer sur un échantillon important. La disparité entre les œuvres peut toutefois être approchée en introduisant l'hypothèse que le nombre d'auteurs différents est un indicateur de cette dimension de la diversité. Plus les individus achèteront des œuvres d'auteurs différents, plus la disparité sera forte, et inversement. De même, distinguer entre les œuvres des éditeurs dominants et ceux plus confidentiels peut également permettre de révéler une certaine disparité dans les genres traités (les seconds se positionnant plus volontiers sur les marchés de niche) ainsi que sur les thèmes abordés.

En matière de diversité culturelle, les deux côtés du marché, l'offre et la demande, présentent leurs spécificités. II convient donc de distinguer la diversité offerte de la diversité consommée et d'analyser dans quelle mesure la diversité offerte correspond à la diversité consommée par les agents économiques. Cette distinction soulève une question cruciale :

\section{Figure 1 - Les trois dimensions de la diversité selon Stirling (1999, 2007)}

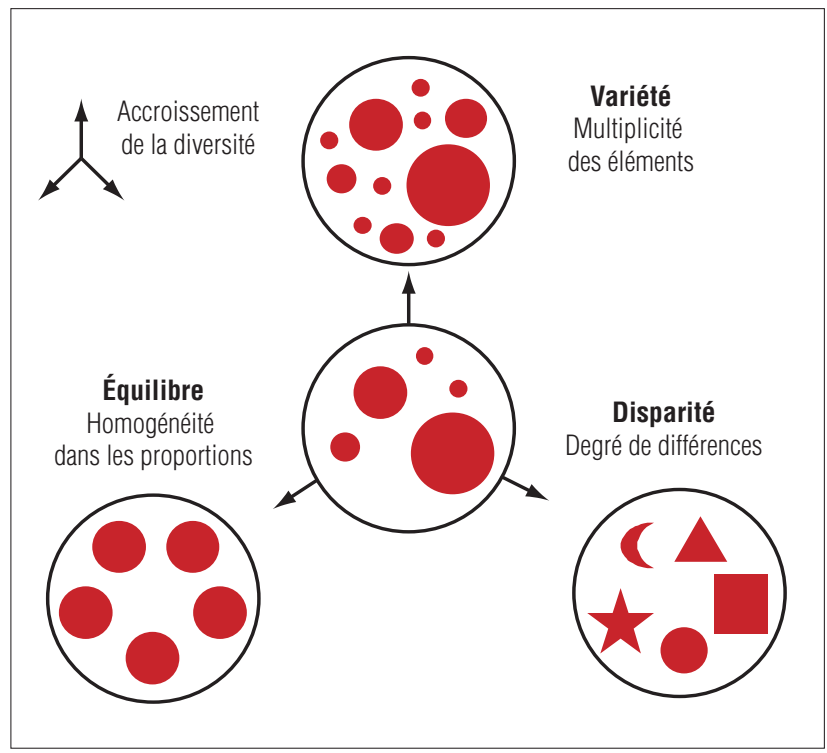

Source : DEPS, Ministère de la Culture et de la Communication, 2011

un accroissement de la diversité offerte a-t-il un impact positif sur la diversité consommée? Autrement dit, avec quelle ampleur diversité offerte et diversité consommée interagissent-elles? Toutefois, comme souligné ci-dessus, les données recueillies par GfK étant des données de ventes, l'analyse portera pour l'essentiel sur la diversité consommée. La diversité offerte ne pouvant être abordée que de manière parcellaire, via la diversité produite (le nombre de nouvelles références mises sur le marché chaque mois).

Au final, les principaux critères retenus, chaque critère étant analysé, si cela s'avère possible et pertinent, par segment (genre) et par circuit de distribution, sont présentés dans le tableau page suivante. Les résultats concernant chacun des indicateurs ne sont toutefois pas nécessairement repris dans cette synthèse. Le lecteur intéressé pourra se reporter au rapport complet pour une étude exhaustive de tous les critères d'analyse de la diversité.

* Cet encadré méthodologique est identique à celui proposé dans le document sur la diversité culturelle dans l'industrie de la musique enregistrée puisque les deux filières ont été étudiées dans le cadre de la même étude (voir Marc BouRREAU, François MOREAU, Pierre SENELLART, la Diversité culturelle dans l'industrie de la musique enregistrée en France (2003-2008), Paris, Ministère de la Culture et de la Communication, DEPS, coll. "Culture études », 2011-5 et M. BouRREAU, M. Gensollen, F. Moreau, S. Peltier et P. Senellart, l'Appréciation et les processus de modification de la diversité dans les filières des industries culturelles, Rapport pour le ministère la Culture et de la Communication, DEPS, 2009, 242 p.). 
Tableau 1 - Les indicateurs de diversité retenus

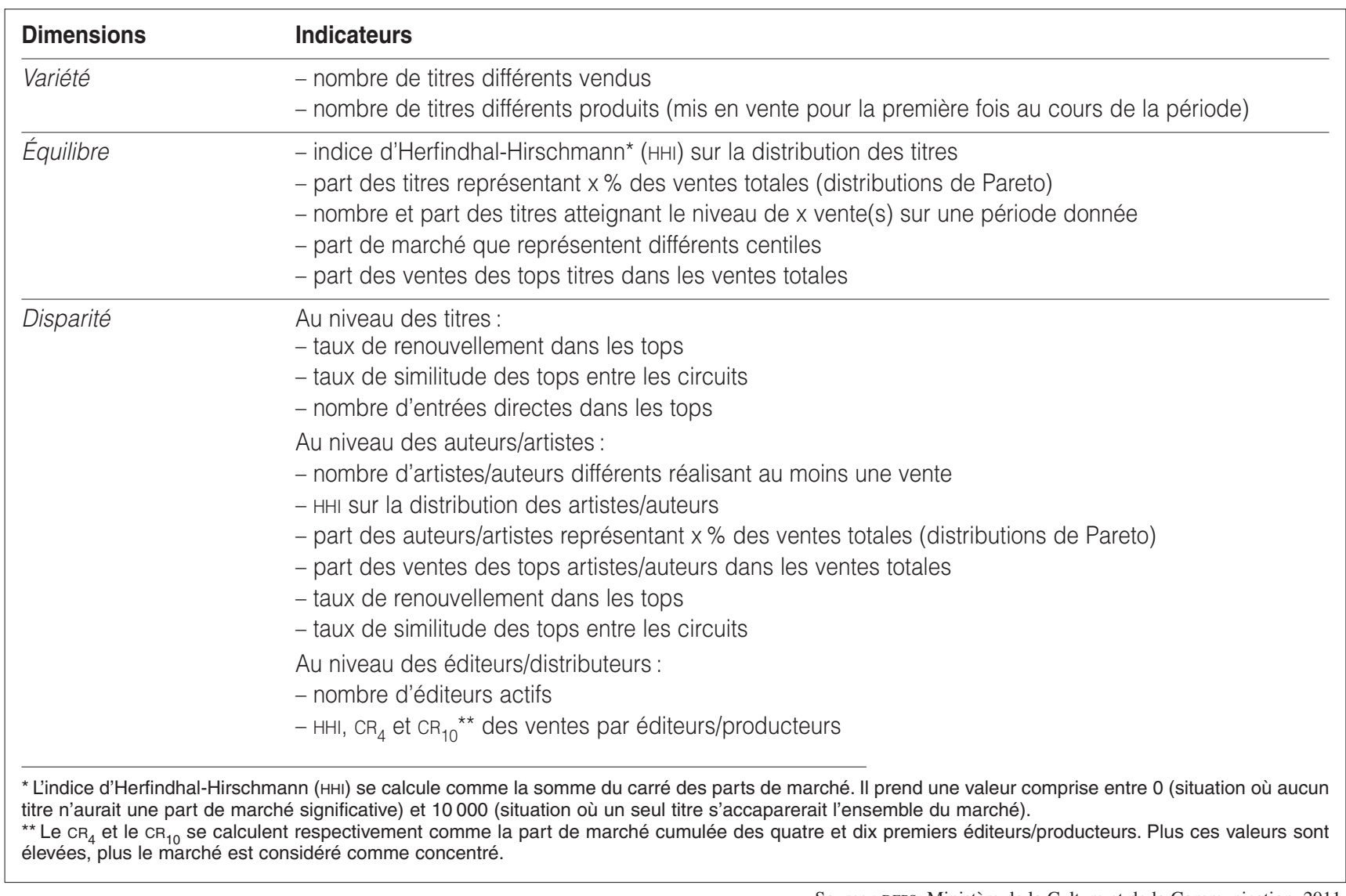

Source : DEPS, Ministère de la Culture et de la Communication, 2011.

\section{RÉSUMÉ}

Si la production éditoriale française a beaucoup progressé au cours des années 2000 en termes de titres et d'exemplaires produits annuellement, comment apprécier si ce bond quantitatif s'accompagne ou non d'une plus grande diversité de la consommation? Fondée sur l'approche tridimensionnelle d'Andrew Stirling, la mesure de la diversité culturelle est analysée sur trois segments éditoriaux (jeunesse, bandes dessinées, littérature) selon trois dimensions : la variété produite et consommée, l'équilibre des ventes entre les différents titres et la disparité des œuvres et des auteurs lus. L'analyse apporte des éléments de réponse à la question de l'efficacité de la loi sur le prix unique en termes de diversité culturelle et sur l'effet de levier des ventes en ligne (hypothèse de la longue traîne).

\section{ABSTRACT}

Although there has been a marked increase in French publishing output throughout the 2000s, in terms both of annual print runs and titles released, how are we to assess whether this growth is also accompanied by increasingly diverse consumption? Based on Andrew Stirling's three-pronged approach, cultural diversity within three different publishing areas (the youth market, comic books and literature) is analysed on three levels: variety produced and consumed, the balance of sales between different titles and the range of works and authors read. This analysis provides some responses to the question of how effective fixed book pricing legislation has been in terms of cultural diversity and the leveraging effect of online sales (the long tail theory).

Tous les documents publiés par le DEPS sont téléchargeables sur http://www.culture.gouv.fr/deps

Le DEPS n'assurant pas de diffusion physique de ses collections, nous vous proposons de vous informer régulièrement des parutions par message électronique. Pour ce faire, merci de bien vouloir nous communiquer votre courriel à l'adresse contact.deps@culture.gouv.fr 